\title{
Temporary Depletion of Microglia during the Early Postnatal Period Induces Lasting Sex-Dependent and Sex-Independent Effects on Behavior in Rats
}

\author{
(D) Jonathan W. VanRyzin, ${ }^{1}{ }^{-}$Stacey J. Yu, ${ }^{2}{ }^{-}$Miguel Perez-Pouchoulen, ${ }^{2}$ and ${ }^{-}$Margaret M. McCarthy ${ }^{1,2}$
}

DOI:http://dx.doi.org/10.1523/ENEURO.0297-16.2016

${ }^{1}$ Program in Neuroscience, University of Maryland School of Medicine, Baltimore, MD 21201, and ${ }^{2}$ Department of Pharmacology, University of Maryland School of Medicine, Baltimore, MD 21201

\begin{abstract}
Microglia are the primary immune cells of the brain and function in multiple ways to facilitate proper brain development. However, our current understanding of how these cells influence the later expression of normal behaviors is lacking. Using the laboratory rat, we administered liposomal clodronate centrally to selectively deplete microglia in the developing postnatal brain. We then assessed a range of developmental, juvenile, and adult behaviors. Liposomal clodronate treatment on postnatal days 0,2 , and 4 depleted microglia with recovery by about 10 days of age and induced a hyperlocomotive phenotype, observable in the second postnatal week. Temporary microglia depletion also increased juvenile locomotion in the open field test and decreased anxietylike behaviors in the open field and elevated plus maze. These same rats displayed reductions in predator odor-induced avoidance behavior, but increased their risk assessment behaviors compared with vehicle-treated controls. In adulthood, postnatal microglia depletion resulted in significant deficits in male-specific sex behaviors. Using factor analysis, we identified two underlying traits-behavioral disinhibition and locomotion-as being significantly altered by postnatal microglia depletion. These findings further implicate microglia as being critically important to the development of juvenile and adult behavior.
\end{abstract}

Key words: behavior; liposomal clodronate; microglia; postnatal development

\section{Significance Statement}

Microglia are critical regulators of postnatal brain development, a time important for the organization of neural architecture and the refinement of synaptic patterning. These immune cells engage in a range of functions, from promoting cell genesis and controlling cell number to facilitating the establishment and maturation of synaptic connections. Despite the current understanding of actions of microglia in the postnatal period, very little is known about how microglia's influences on these early developmental processes affect the expression of behavior later in life. Our findings highlight the importance of microglia during this critical developmental window and suggest the necessity of these cells for the normal expression of juvenile and adult behavior.

\section{Introduction}

Microglia, the brain's primary immune cells, are derived from yolk-sac macrophage precursors that migrate into the brain during early embryonic development, where they

Received October 4, 2016; accepted November 22, 2016; First published November 29, 2016.

The authors declare no conflicts of interest. take up permanent residence (Alliot et al., 1999; Ginhoux et al., 2010; Kierdorf et al., 2013). Throughout development, microglia continue to mature and multiply until they reach their adult numbers and effectively survey the brain 
(Davalos et al., 2005; Nimmerjahn et al., 2005; Schwarz et al., 2012; Nikodemova et al., 2015). Importantly, microglia mature and develop alongside neurons and astrocytes, placing these cells in a unique position to significantly influence the developmental trajectory of the brain.

In early brain development, microglia display an array of functions outside of their normal capacity to maintain homeostasis and respond to injury. Microglia regulate neural precursor populations and cell number by providing trophic support (Morgan et al., 2004; Antony et al., 2011; Ueno et al., 2013; Shigemoto-Mogami et al., 2014), phagocytose dead and dying cells, and actively induce cell death (Marín-Teva et al., 2004; Bessis et al., 2007; Wakselman et al., 2008; Cunningham et al., 2013), a process that continues even in the adult brain (Sierra et al., 2010). Microglia are critical to the proper formation of neural circuitry by "pruning" unnecessary synapses (Stevens et al., 2007; Schafer et al., 2012; Ji et al., 2013), aid in synaptic maturation and function (Tremblay et al., 2010; Paolicelli et al., 2011; Hoshiko et al., 2012; Squarzoni et al., 2014), and mediate the development of sexspecific circuitry (Lenz et al., 2013).

The neural control of normal behaviors also relies heavily on microglia function. Altering microglia function by constitutive genetic deletion of CX3CR1 results in deficient motor learning, spatial memory, and fear conditioning along with impairments in long-term potentiation in adult mice (Rogers et al., 2011). Moreover, these mice display abnormal social behaviors and increased repetitive and self-grooming behavior (Zhan et al., 2014). Genetic microglia depletion in juvenile and adult mice induces impairments in motor learning and memory and reduced fear conditioning (Parkhurst et al., 2013). Similarly, pharmacological attempts to specifically deplete microglia find abnormal spatial memory and social behavior in adult mice; however, behavioral deficits are no longer observed after microglia repopulate the brain (Torres et al., 2016).

These studies advance our understanding of the role microglia play in modulating behavior; however, the conclusions drawn are limited to the period of microglia dysfunction or depletion and do not directly address microglial influence specifically during development. It has been suggested that microglia are most critical for establishing brain architecture and circuitry necessary for normal behavior (Frost and Schafer, 2016), and little research has investigated the influence of microglia on the

This work was funded by NIH Grants R01-MH52716 and R01-DA039062 to M.M.M.

Correspondence should be addressed to either of the following: Jonathan W. VanRyzin, 655 W. Baltimore St., BRB-5014, Baltimore, MD 21201. E-mail: jvanryzin@umaryland.edu; or Margaret M. McCarthy; E-mail: mmccarthy@ som.umaryland.edu.

DOI:http://dx.doi.org/10.1523/ENEURO.0297-16.2016

Copyright (C) 2016 VanRyzin et al.

This is an open-access article distributed under the terms of the Creative Commons Attribution 4.0 International, which permits unrestricted use, distribution and reproduction in any medium provided that the original work is properly attributed. organization of brain and behavior during early periods of development.

To address this issue, we used liposomal clodronate to selectively deplete microglia during the first weeks of postnatal life, a time critical for the continued development of many brain regions, circuitry refinement, and sexual differentiation of the brain. Using male and female rats, we investigated two questions. First, does microglia depletion affect the postnatal developmental trajectory of pups? Second, are there enduring behavioral effects of temporary microglia depletion in juvenile and adult rats? We demonstrate pervasive sex-dependent and sexindependent effects of early postnatal microglia depletion on the later expression of behavior. Together, these data provide new insight into the role of microglia during the early postnatal period in mediating the normal maturation of behavioral circuits in the brain.

\section{Materials and Methods}

\section{Animals}

Adult Sprague-Dawley rats (Charles River Laboratories, Wilmington, MA) were housed in polycarbonate cages (20 $\times 40 \times 20 \mathrm{~cm})$ and maintained on a 12:12-h reverse light/dark cycle with ad libitum food and water. Animals were mated in our facility, and pregnant females were allowed to deliver naturally, with the day of birth designated as postnatal day 0 (PNO). On PNO, pups were sexed, treated, and culled to no more than 12 pups per dam. Both male and female pups were used in this study, with treatment groups and sexes balanced across four litters. All animal procedures were performed in accordance with the University of Maryland Baltimore animal care and use committee's regulations.

\section{Microglia depletion}

On PNO, 2, and 4, liposomal clodronate (LC; Encapsula NanoSciences, Brentwood, TN) or vehicle (VEH) liposomes were administered by bilateral intracerebroventricular (i.c.v.) injection, performed under cryoanesthesia. A 25 -gauge 1- $\mu$ L Hamilton syringe attached to a stereotaxic manipulator was placed $1 \mathrm{~mm}$ caudal to bregma and 1 $\mathrm{mm}$ lateral to the midline. The syringe was lowered $4 \mathrm{~mm}$ into the brain and backed out $1 \mathrm{~mm}$. One microliter of drug or vehicle was infused over $30 \mathrm{~s}$, and the procedure was repeated on the opposite hemisphere. The separation of pups from the dam was kept to a minimum, for a duration of approximately $1 \mathrm{~h}$.

\section{Histology and immunohistochemistry}

Rats of either sex were fatally anesthetized by intraperitoneal injection of Fatal Plus (Vortech Pharmaceuticals, Dearborn, MI) and transcardially perfused with PBS $(0.1 \mathrm{M}$, $\mathrm{pH} 7.4$ ) followed by $4 \%$ paraformaldehyde (PFA; $4 \%$ in PBS, pH 6.8). Brains were removed and postfixed in $4 \%$ PFA for $48 \mathrm{~h}$ at $4^{\circ} \mathrm{C}$, then kept in $30 \%$ sucrose at $4^{\circ} \mathrm{C}$ until fully submerged. Coronal sections were cut into three alternating series at a thickness of $45 \mu \mathrm{m}$ via cryostat (Leica CM3050S) and mounted on silane-coated slides.

Slide-mounted sections from one alternate series were rinsed in Tris-buffered saline (TBS; $0.05 \mathrm{M}, \mathrm{pH}$ 7.6) and 
incubated in $50 \%$ methanol with $0.3 \%$ hydrogen peroxide for $1 \mathrm{~h}$ at room temperature to inhibit endogenous peroxidase activity. Sections were rinsed with TBS again, blocked with $5 \%$ normal goat serum (NGS) in TBS + $0.4 \%$ Triton X-100 (TBS-T), and incubated overnight at room temperature in 5\% NGS in TBS-T containing rabbit polyclonal antibody against ionized calcium binding adapter molecule 1 (Iba1; 1:1000 dilution; Wako Chemicals, Neuss, Germany; cat. \#019-19741, RRID:AB_839504) to label microglia. Subsequently, sections were rinsed in TBS and incubated in 5\% NGS in TBS-T containing biotinylated anti-rabbit secondary antibody (1:500 dilution; Vector Laboratories, Burlingame, CA; cat. \#BA-1000, RRID:AB_2313606) for $1 \mathrm{~h}$ at room temperature, rinsed again in TBS, and incubated with $A B C$ reagent $1: 500$ dilution; Vectastain Elite ABC Kit, Vector Laboratories; cat. \#PK-6100) in TBS-T for $1 \mathrm{~h}$ at room temperature. After further rinsing in TBS, $\mathrm{Iba}^{+}$cells were visualized using nickel-enhanced DAB chromogen [0.05\% 3,3'diaminobenzidine, $0.2 \%$ nickel (II) sulfate, $0.006 \%$ hydrogen peroxide; all from Sigma-Aldrich, St. Louis, MO] in TBS for 1.5-3 min. Finally, sections were rinsed in TBS, counterstained with hematoxylin (Vector Laboratories; cat. \#H-3401) according to the manufacturer's protocol, cleared with ascending alcohol treatment, defatted in $x y-$ lene, and coverslipped in DPX mounting medium.

\section{Image analysis and quantification}

Immunolabeled sections were imaged using a Nikon Eclipse E600 microscope equipped with an MBF Bioscience CX9000 digital camera and analyzed using NIH ImageJ software. Modifications to image brightness and contrast were performed in ImageJ. For each brain region analyzed, four to six images were taken from two to three sections per rat. Regions of interest were drawn for each image, and the number of $\mathrm{Iba} 1^{+}$cells was counted. The Iba $1^{+}$cell number was normalized to the area of each region of interest to yield an Iba $1^{+}$cell density. Finally, the lba $1^{+}$cell density was averaged across images to generate an average density value for each rat in each brain region. Image acquisition and analysis was performed by an experimenter blind to treatment condition.

\section{Behavioral testing}

All behavioral testing was performed during the rat's dark phase of the light cycle $(\sim 2 \mathrm{~h}$ after the start of the dark phase) and under red light illumination. Behavioral tests were performed and scored offline (unless stated otherwise) by two experimenters blind to treatment condition.

\section{Developmental behavioral testing}

Assessment of developmental behaviors began on PN5 and continued until PN15. Specific behaviors were assessed each day over the indicated age range for each rat pup.

\section{Surface righting reflex (PN5-PN10)}

Each pup was placed on its back on a flat surface. The time for the pup to return to its four limbs was recorded. The cutoff time for this test was $30 \mathrm{~s}$.

\section{Cliff aversion (PN5-PN10)}

Each pup was placed on a flat surface with its snout and forepaws hanging over an edge. The time for the pup to completely retract from the edge was recorded, up to $30 \mathrm{~s}$.

\section{Wire suspension (PN10-PN14)}

The pup's forepaws were placed against a horizontal wire rod suspended above a padded bin. The duration of time each pup hung from the wire was recorded, up to $10 \mathrm{~s}$.

\section{Negative geotaxis (PN5-PN14)}

Each pup was placed facedown on a ramp with a 25-degree incline, covered with a wire mesh to enable traction. The time for the pup to turn 180 degrees with its head and trunk oriented to the top of the incline was recorded, up to $30 \mathrm{~s}$.

\section{Locomotion (PN5-PN14)}

Each pup was placed on a flat surface in the center of an underlaid circle (13-cm diameter). The time for all four legs of the pup to exit the circle completely was recorded, up to $30 \mathrm{~s}$.

\section{Nest seeking (PN5-PN15)}

Rat pups were placed in the center of a rectangular arena $(20 \times 12 \mathrm{~cm})$ between two goal containers filled with equal amounts of bedding, one containing fresh bedding and one containing bedding taken from their home nest. Four centimeters from either goal, the arena was marked to determine the "final side choice" of each pup. This measure was scored to account for pups that failed to reach either goal before the test duration expired. At the start of each trial, the pup was placed facing 90 degrees from either arm. The first orientation of the pup (toward or away from home bedding), the final side choice of the pup, and the goal container reached were recorded. Pup activity toward the nest bedding goal was given a positive score, whereas activity toward the clean bedding goal was given a negative score. Once the pup's head and forepaws crossed into either goal (or reaching a cutoff time of $120 \mathrm{~s}$ ), the pup was removed from the arena. Each pup was tested in two trials per day (intertrial interval of 30 s), and the pup's starting position was counterbalanced between trials. The total score for each trial was calculated and averaged between the two trials, resulting in one score per pup, per day.

\section{Maternal isolation-induced ultrasonic vocalization re- cording (PN8)}

Ultrasonic vocalizations (USVs) were recorded for $3 \mathrm{~min}$ from rat pups that were isolated from maternal interaction. Ambient temperature was maintained at $22^{\circ} \mathrm{C} \pm 1^{\circ} \mathrm{C}$. On the day of testing, pups were placed individually into a sound-attenuated recording chamber $(15 \times 15 \mathrm{~cm})$ and recorded for 3 min using an UltraSoundGate Condenser Microphone (CM16; Avisoft Bioacoustics, Glienicke, Germany), placed at a fixed height $(10 \mathrm{~cm})$ above the pups. The microphone was connected to a personal computer via an UltraSoundGate $116 \mathrm{Hb}$ recording interface (Avisoft Bioacoustics). Acoustic data were recorded at a sampling rate of $250,000 \mathrm{~Hz}$ in 16 -bit format and analyzed using 
SASLab Pro (version 5.10; Avisoft Bioacoustics). Before analysis, recordings were processed with a fast Fourier transform (512 FFT, 75\% frame size, flattop window, and $93.75 \%$ time window overlap). A high-pass filter was used to eliminate background noise below $10 \mathrm{kHz}$. USVs were automatically detected using a threshold-based algorithm (-35-dB threshold, 20-ms hold time) and confirmed by manual inspection to ensure that all calls were appropriately detected. Analysis was performed by an experimenter blind to the condition and sex of the pups.

\section{Juvenile behavioral testing}

\section{Open field test (PN25)}

Rats were placed in an open polycarbonate arena $(78 \times$ $78 \mathrm{~cm}, 40 \mathrm{~cm}$ high) for $10 \mathrm{~min}$. The floor was underlaid with a grid delineated into a center and perimeter regions. The time spent in the center grid squares and the number of grid crosses was recorded.

\section{Spontaneous alternations (PN25-PN29)}

Spontaneous alternation was tested using a black polycarbonate T-maze consisting of an entry arm $(50 \times 16 \mathrm{~cm}$, $30 \mathrm{~cm}$ high) connected to a right and left arm $(50 \times 10 \mathrm{~cm}$, $30 \mathrm{~cm}$ high). The right and left arms were split by a short divider that extended $10 \mathrm{~cm}$ into the entry arm to prevent the animal from simultaneously surveying both arms before entering. Rats were placed in the entry arm of the T-maze and allowed to explore until they completely entered either the right or left arm (sample phase). Upon entering an arm, a barrier was placed to confine rats to that arm for $30 \mathrm{~s}$. Rats were removed and placed back into the entry arm and allowed to choose between the two arms again (test phase). The latency to enter an arm on the sample and test phases was recorded, as well as the chosen arm. Successful alternation was scored if the chosen test arm was opposite the chosen sample arm. Rats completed two trials (each trial comprised of a sample and test phase) on average each day (intertrial interval $10 \mathrm{~min}$ ), and the total number of successful alternations was calculated as a percent of total number of trials $(8-10$ total trials per rat).

\section{Novel object recognition (PN26-PN28)}

On PN26, rats were placed into the same arena used for the open field test for $10 \mathrm{~min}$ to allow for continued habituation to the arena. On PN27, rats were exposed to a pair of identical objects (object $A+A$; sampling trial) placed on opposite ends of the arena for $5 \mathrm{~min}$. After the initial exposure, animals were placed back in their home cages for $1 \mathrm{~h}$, after which they were returned to the arena and exposed to the now familiar object and a novel object (object A + B). On PN28, animals were tested for their object memory $24 \mathrm{~h}$ after the first sampling trial, with a second novel object (object $A+C$ ). The time spent investigating each object was recorded, and the recognition index was calculated (time with novel object/time with novel object + time with familiar object). The position of objects in the arena and the order of object exposure was counterbalanced between treatment groups.

\section{Social recognition (PN30)}

Rats were singly housed in a test cage (identical to their home cage) for $2 \mathrm{~h}$ before the start of the test to allow for habituation. While singly housed, rats had ad libitum access to food and water. After $2 \mathrm{~h}$, a stimulus rat (age PN15-21) was placed into the test cage with the test rat for $4 \mathrm{~min}$. The stimulus rat was then removed, and the test rat remained in the test cage for a retention interval of $30 \mathrm{~min}$. At this point, the original (now familiar) stimulus rat and a novel stimulus rat were placed in the test cage with the test rat. The time spent investigating the stimulus rat during the sample phase was recorded. During the recognition phase, the time spent investigating each of the stimulus rats was recorded, and recognition index was calculated (time with novel rat/time with novel rat + time with familiar rat).

\section{Elevated plus maze (PN32)}

Rats were placed in the center of a black polycarbonate elevated plus maze (elevated $72 \mathrm{~cm}$ from the ground) consisting of two opposing open $(102.5 \times 12 \mathrm{~cm})$ and closed $(102.5 \times 12 \mathrm{~cm}, 45.5 \mathrm{~cm}$ high) arms and allowed to freely explore the maze for $5 \mathrm{~min}$. The time spent in the open arms of the maze was recorded, and the percentage of the test duration spent in the open arms was calculated.

\section{Predator odor exposure (PN33)}

On PN32, rats were placed into the testing arena for 10 min to allow for habituation. The testing arena was divided into two compartments: a large main compartment $(40 \times$ $27 \mathrm{~cm}, 30 \mathrm{~cm}$ high) and a smaller rear compartment $(20 \times$ $27 \mathrm{~cm}, 30 \mathrm{~cm}$ high). An opaque polycarbonate barrier divided the two compartments but contained an opening $(6 \mathrm{~cm}$ wide $\times 6 \mathrm{~cm}$ high) through which rats were able to pass. The purpose of this barrier was to provide an area in which the rats would be able to hide from the cat odor stimulus and allow for the expression of risk-assessing behaviors. Cat-scented pieces of cloth were prepared by placing the pieces in the bed of an adult male cat for $48 \mathrm{~h}$ before testing. On PN33, rats were tested in two consecutive phases: a baseline phase (2.5 min), during which the rats were exposed to an unscented piece of cloth $(10 \times 5$ $\mathrm{cm}$ ) folded in half lengthwise and placed in a large binder clip. The clip was placed at the far end of the large compartment (opposite the small compartment). At the end of the baseline phase, the unscented cloth square was replaced with a cat-scented cloth of similar dimensions, and the behavioral test continued for an additional $5 \mathrm{~min}$. In both test phases, the time spent behind the barrier, duration and number of interactions with the cloth (defined as the touching of the cloth or binder clip with the nose or whiskers), duration and number of freezing bouts (defined as hunched posture with a complete absence of motion), duration and number of stretched-attending bouts (defined stretched, low-body posture with deliberate orientation and attention to the cloth square, while all locomotion is absent), and duration and number of stretched locomotor bouts (defined as stretched, lowbody posture maintained while moving around the arena) were recorded. Data were normalized to a 5-min total duration for each phase of the test.

\section{Adult sex behavior testing}

Female sex behavior (PN60+)

Intact and naturally cycling female rats were tested for the expression of female proceptive behaviors and lordo- 
sis in response to an actively mounting male rat. Females were tested daily throughout their estrus cycle for $20 \mathrm{~min}$ each day, and behaviors were scored for each rat on the day of pro-estrus (determined by vaginal cytology). Proceptive behaviors (number of hops and darts, solicitations) and lordoses were recorded, and a lordosis quotient was calculated (number of lordoses/number of successful mounts). Hops were defined as a short jump with the female landing on all four paws in a crouched position. Darts were defined as a short run with the female abruptly stopping in a crouched position. Solicitations were defined as the head-wise orientation toward the male followed by an abrupt runaway into a presenting posture. Behavior was scored live by an experimenter blind to the animal's treatment.

\section{Male sex behavior (PN60+)}

Intact male rats were tested for male copulatory behaviors in response to a hormonally primed stimulus female rat. Males were tested once a week for 2 weeks in a 20-min behavioral test. Stimulus females were hormonally primed after being given $10 \mu \mathrm{g}$ of estradiol benzoate in 0.1 $\mathrm{ml}$ sesame oil subcutaneously 1 and 2 days before testing, and $500 \mu \mathrm{g}$ of progesterone in $0.1 \mathrm{ml}$ sesame oil subcutaneously $6 \mathrm{~h}$ before testing. The number of mounts, intromission-like behaviors, and ejaculatory behaviors were recorded, as well as the latency to the first observation of each behavior. If none of these behaviors were observed, the latency was set equal to the test duration (1200 s). A mount was counted when the male mounted the female from the rear, placing both forepaws on the flanks of the female and thrusting against her. Intromission-like behaviors were counted when the male performed a mount, but during the thrusts quickly shifted his weight, engaged in extreme flexions of his haunches, and reflexively disengaged. Ejaculatory behaviors were defined as intromission-like behaviors that did not reflexively disengage, but instead the male remained in a hunched posture after thrusting. This was accompanied by a period of genital grooming followed by disinterest in the female. Behavior was scored live by an experimenter blind to the animal's treatment.

\section{Statistical analysis}

Statistical analysis was performed using $R$ ( $R$ Core Team, 2016; version 3.2.1). All data were initially analyzed for a main effect of sex by two- or three-way ANOVA when appropriate. A main effect of sex was detected only for body weight. Therefore all other data were collapsed and analyzed independent of sex, with the exception of sex behavior, which was analyzed separately for males and females. Analysis proceeded using either Welch's $t$-test or two-way ANOVA (repeated measures when age or test phase were a factor) when appropriate. In some cases, repeated-measures tests violated the assumption of sphericity; when this occurred, the Greenhouse-Geisser correction was applied (corrected $p$ values are listed in Table 1). If a significant interaction was detected, post hoc pairwise comparisons were performed using the Holm's sequential Bonferroni correction. Adjusted $\alpha$ values for post hoc tests are presented next to the calculated $p$ value in Table 1. We chose a priori to analyze male sex behavior with a pairwise comparison on the second test day, as male performance improves with experience. The data for all male sex behaviors and female lordosis quotient did not follow a normal distribution and were therefore analyzed by a Wilcoxon rank sum test. Cohen's $d, \eta^{2}$, and Hodges-Lehmann $(\mathrm{HL})$ estimates were calculated as measures of effect size for $t$-tests, ANOVAs, and Wilcoxon rank sum tests, respectively. Specific values for test statistics, $p$ values, effect size, and power are listed in Table 1, and are referenced in text as superscript letters. Analyses were considered significant when $p \leq 0.05$, unless otherwise noted in Table 1.

Behavioral data for factor analysis were normalized by log transformation before analysis. Only subjects that had complete behavioral datasets were included in the factor analysis ( $n=16$ for VEH, $n=13$ for LC). Factor analysis was conducted with varimax rotation with a factor-loading cutoff of 0.3. The resulting factors were retained if their eigenvalue was greater than 1 (based on the Kaiser criterion), generating a four-factor solution for our dataset. Individual factor scores were calculated for each subject, and each factor was analyzed independently by Welch's $t$-test.

\section{Results}

\section{Liposomal clodronate treatment reduces microglia number and alters brain morphology}

To determine the extent of microglia depletion and the time course of microglia recovery, we injected male and female rat pups i.c.v. with either LC or VEH liposomes on PNO, 2, and 4 (Fig. 1) and compared brain sections at PN5, 10, and 15. Gross morphological examination showed disruptions in overall brain morphology, most notably characterized by an enlargement of the ventricles and a thinning of the cortex. These effects were apparent as early as PN10 and highly variable between individual pups. In some cases, brain specimens were extremely fragile, making analysis from these individuals difficult. Examination of Nissl-stained sections revealed the presence of major brain regions that in extreme cases appeared displaced and compressed around the ventricles.

We visualized microglia by immunohistochemistry using an antibody against the microglia/macrophage specific marker Iba1. Iba1 ${ }^{+}$cell density was quantified in various brain regions on PN5 (24 h after last LC injection). Analysis revealed significantly fewer lba $1^{+}$cells in the hippocampus (Fig. $2 a-c ; p=0.011^{a}$ ) and the amygdala (Fig. $2 g-i ; p=$ $\left.0.005^{\mathrm{b}}\right)$, with a strong trend toward significant reduction in the cortex (Fig. $2 d-f ; p=0.054^{\circ}$ ) and the hypothalamus (Fig. $\left.2 j-l ; p=0.059^{d}\right)$. Overall, the effect of depletion ranged between $40 \%$ and $60 \%$ in the regions analyzed.

Limited quantification found that by $\mathrm{PN} 10, \mathrm{Iba} 1^{+}$cells from LC-treated pups robustly repopulated all regions of the brain ${ }^{\mathrm{e}} \mathrm{h}$ (Fig. 2c, $f, i$, ). In some cases, this repopulation resulted in a greater number of microglia compared to VEH-treated controls ( $p=0.036$ for PN10 amygdala; Fig. 2i). The number of Iba1 ${ }^{+}$cells in LC-treated pups normalized by PN15, as sections from VEH- and LC-treated pups displayed comparable densities of $\mathrm{Iba}^{+}$cells across brain regions ${ }^{i-1}$ (Fig. $\left.2 c, f, i, l\right)$. Thus, the clodronate 
Table 1. Summary of statistical analyses.

\begin{tabular}{|c|c|c|c|c|c|c|c|}
\hline & & & & & & & Power or \\
\hline Line & Data structure & Type of test & Description of analysis & Test value & $p$-value & Effect size & $95 \% \mathrm{Cl}$ \\
\hline $\mathrm{a}$ & Normal distribution & Welch's $t$-test & PN5 hippocampus: VEH vs. LC & $t_{((2.9847)}=5.667$ & 0.011 & $d=4.625$ & 0.984 \\
\hline b & Normal distribution & Welch's t-test & PN5 amygdala: VEH vs. LC & $t_{(3.7237)}=5.996$ & 0.005 & $d=2.38$ & 0.596 \\
\hline c & Normal distribution & Welch's t-test & PN5 cortex: VEH vs. LC & $t_{(3.3363)}=2.915$ & 0.054 & $d=4.896$ & 0.991 \\
\hline d & Normal distribution & Welch's t-test & PN5 hypothalamus: VEH vs. LC & $t_{(3.7531)}=2.108$ & 0.059 & $d=3.064$ & 0.798 \\
\hline e & Normal distribution & Welch's t-test & PN10 hippocampus: VEH vs. LC & $t_{(1.0548)}=-0.875$ & 0.536 & $d=1.045$ & 0.13 \\
\hline$f$ & Normal distribution & Welch's t-test & PN10 amygdala: VEH vs. LC & $t_{(2.6755)}=-3.941$ & 0.036 & $d=2.461$ & 0.457 \\
\hline g & Normal distribution & Welch's t-test & PN10 cortex: VEH vs. LC & $t_{(1.0034)}=-2.013$ & 0.293 & $d=3.409$ & 0.702 \\
\hline $\mathrm{h}$ & Normal distribution & Welch's t-test & PN10 hypothalamus: VEH vs. LC & $t_{(1.196)}=-1.257$ & 0.401 & $d=1.42$ & 0.197 \\
\hline i & Normal distribution & Welch's t-test & PN15 hippocampus: VEH vs. LC & $t_{(1.0067)}=-1.248$ & 0.429 & $d=1.248$ & 0.22 \\
\hline j & Normal distribution & Welch's t-test & PN15 amygdala: VEH vs. LC & $t_{(1.1786)}=-1.437$ & 0.36 & $d=1.269$ & 0.225 \\
\hline $\mathrm{k}$ & Normal distribution & Welch's t-test & PN15 cortex: VEH vs. LC & $t_{(1.312)}=-1.269$ & 0.384 & $d=1.437$ & 0.274 \\
\hline । & Normal distribution & Welch's t-test & PN15 hypothalamus: VEH vs. LC & $t_{(1.0299)}=0.254$ & 0.841 & $d=0.254$ & 0.057 \\
\hline \multicolumn{8}{|c|}{ Body weight } \\
\hline $\mathrm{m}$ & Normal distribution & 3-way ANOVA & Main effect: sex & $F_{(1,29)}=236.343$ & $<0.001$ & $\eta^{2}=0.068$ & 1 \\
\hline \multicolumn{8}{|c|}{ Female body weight } \\
\hline$n$ & Normal distribution & 2-way ANOVA & Main effect: treatment & $F_{(1,14)}=0.077$ & 0.785 & $\eta^{2}=0.072$ & 0.058 \\
\hline o & Normal distribution & 2-way ANOVA & Main effect: age & $F_{(1.146,16.037)}=617.607$ & $<0.001$ & $\eta^{2}=0.974$ & 1 \\
\hline $\mathrm{p}$ & Normal distribution & 2-way ANOVA & Interaction: treatment $\times$ age & $F_{(1.146,16.07)}=2.842$ & 0.108 & $\eta^{2}=0.004$ & 0.375 \\
\hline \multicolumn{8}{|c|}{ Male body weight } \\
\hline q & Normal distribution & 2-way ANOVA & Main effect: treatment & $F_{(1,15)}=6.045$ & 0.027 & $\eta^{2}=0.287$ & 0.633 \\
\hline$r$ & Normal distribution & 2-way ANOVA & Main effect: age & $F_{(1.626,24.387)}=2222.172$ & $<0.001$ & $\eta^{2}=0.992$ & 1 \\
\hline s & Normal distribution & 2-way ANOVA & Interaction: treatment $\times$ age & $F_{(1,626,24.387)}=2.019$ & 0.161 & $\eta^{2}=0.001$ & 0.341 \\
\hline \multicolumn{8}{|c|}{ Cliff aversion } \\
\hline t & Normal distribution & 3-way ANOVA & Main effect: sex & $F_{(1,34)}=1.165$ & 0.288 & $\eta^{2}=0.033$ & 0.183 \\
\hline u & Normal distribution & 2-way ANOVA & Main effect: treatment & $F_{(1,36)}=0.381$ & 0.541 & $\eta^{2}=0.011$ & 0.092 \\
\hline v & Normal distribution & 2-way ANOVA & Main effect: age & $F_{(5,180)}=9.473$ & $<0.001$ & $\eta^{2}=0.155$ & 1 \\
\hline w & Normal distribution & 2-way ANOVA & Interaction: treatment $\times$ age & $F_{(5,180)}=1.460$ & 0.24 & $\eta^{2}=0.031$ & 0.263 \\
\hline \multicolumn{8}{|c|}{ Surface righting } \\
\hline $\mathrm{x}$ & Normal distribution & 3-way ANOVA & Main effect: sex & $F_{(1,34)}=0.177$ & 0.676 & $\eta^{2}=0.004$ & 0.069 \\
\hline y & Normal distribution & 2-way ANOVA & Main effect: treatment & $F_{(1,36)}=9.999$ & 0.003 & $\eta^{2}=0.217$ & 0.868 \\
\hline z & Normal distribution & 2-way ANOVA & Main effect: age & $F_{(5,180)}=3.973$ & 0.014 & $\eta^{2}=0.094$ & 0.944 \\
\hline aa & Normal distribution & 2-way ANOVA & Interaction: treatment $\times$ age & $F_{(5,180)}=2.134$ & 0.11 & $\eta^{2}=0.051$ & 0.489 \\
\hline \multicolumn{8}{|c|}{ Wire hang } \\
\hline $\mathrm{bb}$ & Normal distribution & 3-way ANOVA & Main effect: sex & $F_{(1,34)}=0.934$ & 0.34 & $\eta^{2}=0.015$ & 0.156 \\
\hline cc & Normal distribution & 2-way ANOVA & Main effect: treatment & $F_{(1,36)}=25.742$ & $<0.001$ & $\eta^{2}=0.083$ & 0.999 \\
\hline dd & Normal distribution & 2-way ANOVA & Main effect: age & $F_{(4,144)}=32.229$ & $<0.001$ & $\eta^{2}=0.367$ & 1 \\
\hline ee & Normal distribution & 2-way ANOVA & Interaction: treatment $\times$ age & $F_{(4,144)}=2.223$ & 0.087 & $\eta^{2}=0.032$ & 0.561 \\
\hline \multicolumn{8}{|c|}{ Negative geotaxis } \\
\hline $\mathrm{ff}$ & Normal distribution & 3-way ANOVA & Main effect: sex & $F_{(1,34)}=0.003$ & 0.955 & $\eta^{2}<0.000$ & 0.05 \\
\hline gg & Normal distribution & 2-way ANOVA & Main effect: treatment & $F_{(1,36)}=12.68$ & 0.001 & $\eta^{2}=0.024$ & 0.934 \\
\hline hh & Normal distribution & 2-way ANOVA & Main effect: age & $F_{(9,324)}=44.323$ & $<0.001$ & $\eta^{2}=0.493$ & 1 \\
\hline ii & Normal distribution & 2-way ANOVA & Interaction: treatment $\times$ age & $F_{(9,324)}=1.228$ & 0.291 & $\eta^{2}=0.015$ & 0.492 \\
\hline Loco & & & & & & & \\
\hline jj & Normal distribution & 3-way ANOVA & Main effect: sex & $F_{(1,34)}=1.109$ & 0.3 & $\eta^{2}=0.008$ & 0.176 \\
\hline kk & Normal distribution & 2-way ANOVA & Main effect: treatment & $F_{(1,36)}=93.876$ & $<0.001$ & $\eta^{2}=0.723$ & 1 \\
\hline$\|$ & Normal distribution & 2-way ANOVA & Main effect: age & $F_{(9,324)}=26.712$ & $<0.001$ & $\eta^{2}=0.378$ & 1 \\
\hline $\mathrm{mm}$ & Normal distribution & 2-way ANOVA & Interaction: treatment $\times$ age & $F_{(9,324)}=7.873$ & $<0.001$ & $\eta^{2}=0.088$ & 1 \\
\hline $\mathrm{nn}$ & Normal distribution & Welch's t-test & Post hoc: PN5 LC vs. VEH & $t_{(29.15)}^{(9,324)}=-0.398$ & $0.694 ; \alpha=0.05$ & $d=0.125$ & 0.066 \\
\hline oo & Normal distribution & Welch's t-test & Post hoc: PN6 LC vs. VEH & $t_{(34.126)}=0.766$ & $0.449 ; \alpha=0.025$ & $d=0.25$ & 0.117 \\
\hline $\mathrm{pp}$ & Normal distribution & Welch's t-test & Post hoc: PN7 LC vs. VEH & $t_{(24.031)}=1.869$ & $0.074 ; \alpha=0.0125$ & $d=0.628$ & 0.469 \\
\hline qq & Normal distribution & Welch's t-test & Post hoc: PN8 LC vs. VEH & $t_{(34.211)}=1.888$ & $0.068 ; \alpha=0.01$ & $d=0.618$ & 0.456 \\
\hline rr & Normal distribution & Welch's t-test & Post hoc: PN9 LC vs. VEH & $t_{(34.971)}=1.514$ & $0.139 ; \alpha=0.01667$ & $d=0.494$ & 0.315 \\
\hline ss & Normal distribution & Welch's $t$-test & Post hoc: PN10 LC vs. VEH & $t_{(34.907)}=4.212$ & $<0.001 ; \alpha=0.00714$ & $d=1.374$ & 0.984 \\
\hline $\mathrm{tt}$ & Normal distribution & Welch's $t$-test & Post hoc: PN11 LC vs. VEH & $t_{(35.873)}=4.639$ & $<0.001 ; \alpha=0.0625$ & $d=1.503$ & 0.994 \\
\hline uu & Normal distribution & Welch's $t$-test & Post hoc: PN12 LC vs. VEH & $t_{(31.565)}=3.096$ & $0.004 ; \alpha=0.0083$ & $d=1.021$ & 0.864 \\
\hline vv & Normal distribution & Welch's $t$-test & Post hoc: PN13 LC vs. VEH & $t_{(35.123)}=5.448$ & $<0.001 ; \alpha=0.0056$ & $d=1.745$ & 0.999 \\
\hline ww & Normal distribution & Welch's $t$-test & Post hoc: PN14 LC vs. VEH & $t_{(35.301)}=8.084$ & $<0.001 ; \alpha=0.005$ & $d=2.631$ & 1 \\
\hline Nest & & & & & & & \\
\hline$x x$ & Normal distribution & 2-way ANOVA & Main effect: sex & $F_{(1,34)}=0.0002$ & 0.989 & $\eta^{2}<0.000$ & 0.05 \\
\hline yy & Normal distribution & Welch's $t$-test & VEH vs. LC & $t_{(34.052)}=3.927$ & $<0.001$ & $d=1.285$ & 0.97 \\
\hline Nest & & & & & & & \\
\hline$z z$ & Normal distribution & 3-way ANOVA & Main effect: sex & $F_{(1,34)}=0.015$ & 0.904 & $\eta^{2}<0.000$ & 0.05 \\
\hline aaa & Normal distribution & 2-way ANOVA & Main effect: treatment & $F_{(1,36)}=15.626$ & $<0.001$ & $\eta^{2}=0.043$ & 0.97 \\
\hline bbb & Normal distribution & 2-way ANOVA & Main effect: age & $F_{(10,360)}=2.423$ & 0.008 & $\eta^{2}=0.053$ & 0.943 \\
\hline $\operatorname{ccc}$ & Normal distribution & 2-way ANOVA & Interaction: treatment $\times$ age & $F_{(10,360)}=1.087$ & 0.371 & $\eta^{2}=0.028$ & 0.46 \\
\hline Nest & & & & & & & \\
\hline ddd & Normal distribution & 3-way ANOVA & Main effect: sex & $F_{(1,34)}=0.278$ & 0.602 & $\eta^{2}=0.008$ & 0.081 \\
\hline eee & Normal distribution & 2-way ANOVA & Main effect: age & $F_{(10,360)}=47.962$ & $<0.001$ & $\eta^{2}=0.458$ & 1 \\
\hline fff & Normal distribution & 2-way ANOVA & Main effect: treatment & $F_{(1,36)}=0.075$ & 0.786 & $\eta^{2}=0.002$ & 0.058 \\
\hline $\begin{array}{l}\text { ggg } \\
\text { Isolat }\end{array}$ & Normal distribution & 2-way ANOVA & Interaction: treatment $\times$ age & $F_{(10,360)}=0.369$ & 0.847 & $\eta^{2}=0.004$ & 0.137 \\
\hline hhh & Normal distribution & 2-way ANOVA & Main effect: sex & $F_{(1,23)}=0.253$ & 0.62 & $\eta^{2}=0.004$ & 0.077 \\
\hline iii & Normal distribution & Welch's $t$-test & VEH vs. LC & $t_{(23.928)}=7.337$ & $<0.001$ & $d=2.795$ & 0.999 \\
\hline Spon & & & & & & & \\
\hline $\mathrm{jjj}$ & Normal distribution & 2-way ANOVA & Main effect: sex & $F_{(1,34)}=1.802$ & 0.188 & $\eta^{2}=0.043$ & 0.257 \\
\hline kkk & Normal distribution & Welch's $t$-test & VEH vs. LC & $t_{(33.907)}=2.327$ & 0.026 & $d=0.762$ & 0.626 \\
\hline
\end{tabular}


Table 1. Continued

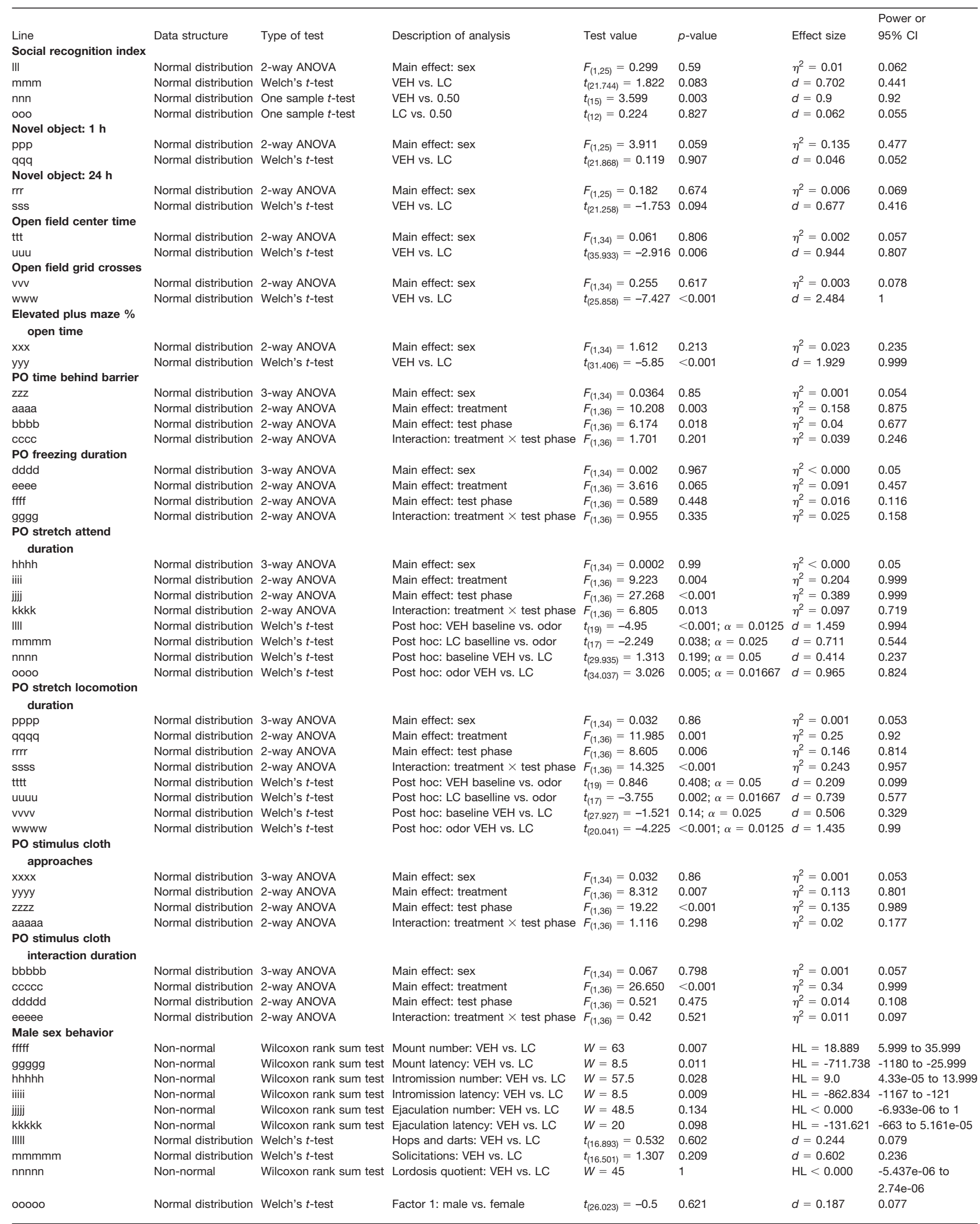


Table 1. Continued

\begin{tabular}{|c|c|c|c|c|c|c|c|}
\hline Line & Data structure & Type of test & Description of analysis & Test value & $p$-value & Effect size & $\begin{array}{l}\text { Power or } \\
95 \% \mathrm{Cl}\end{array}$ \\
\hline ppppp & Normal distribution & Welch's $t$-test & Factor 2: male vs. female & $t_{(18.074)}=-0.691$ & 0.499 & $d=0.263$ & 0.105 \\
\hline qqqqq & Normal distribution & Welch's $t$-test & Factor 3: male vs. female & $t_{(20.761)}=0.704$ & 0.49 & $d=0.256$ & 0.102 \\
\hline rrrrr & Normal distribution & Welch's $t$-test & Factor 4: male vs. female & $t_{(26.982)}=-0.572$ & 0.572 & $d=0.212$ & 0.085 \\
\hline sssss & Normal distribution & Welch's $t$-test & Factor 1: VEH vs. LC & $t_{(26.941)}=-3.267$ & 0.003 & $d=1.187$ & 0.865 \\
\hline $\mathrm{ttttt}$ & Normal distribution & Welch's $t$-test & Factor 2: VEH vs. LC & $t_{(25.663)}=-4.063$ & $<0.001$ & $d=1.449$ & 0.962 \\
\hline unuuu & Normal distribution & Welch's $t$-test & Factor 3: VEH vs. LC & $t_{(25.689)}=1.559$ & 0.131 & $d=0.556$ & 0.301 \\
\hline vvvvv & Normal distribution & Welch's $t$-test & Factor 4: VEH vs. LC & $t_{(21.724)}=1.415$ & 0.171 & $d=0.545$ & 0.291 \\
\hline
\end{tabular}

treatment paradigm resulted in a transient depletion of $\sim 50 \%$ of the microglia population throughout the brain during the first postnatal week, with rapid growth and repopulation that was complete by the end of the second postnatal week.

\section{Body weight throughout the lifespan}

We initially performed three-way repeated-measures ANOVA to examine the effects of sex and treatment on body weight throughout the animal's life and found a significant effect of sex $\left(p<0.001^{\mathrm{m}}\right)$. This prompted us to repeat the analysis as two-way repeated-measures ANOVAs to assess the effect of treatment separately in male ${ }^{\mathrm{q}-\mathrm{s}}$ and female ${ }^{n-p}$ rats. Body weight increased with age for both male and female rats $(p<0.001$ for females, Fig. $3 a ; p<0.001$ for males, Fig. 3b). Interestingly, LC-treated male rats consistently weighed less across the lifespan (main effect of treatment, $p<0.001$ ), whereas LC-treated females did not differ in their weight compared to VEH-treated controls.

\section{Neonatal reflexes and behaviors}

We did not detect an effect of sex on any of the developmental or juvenile behaviors examined, so we collapsed the data across sex and reanalyzed.

\section{Cliff aversion}

Latency to retreat for both VEH- and LC-treated pups improved with age (main effect of age, $p<0.001^{\text {t-w; }}$ Fig. $3 c$ ).

\section{Surface righting}

LC-treated pups consistently righted themselves more quickly than VEH-treated controls (main effect of treatment, $p=0.003$ ), and both groups improved with age (main effect of age, $p=0.014$; Fig. $3 d$ ). ${ }^{x-a a}$

\section{Wire hang}

LC-treated pups hung for significantly less time than VEH-treated controls (main effect of treatment, $p<0.001$; Fig. 3e), and hang time increased with age for all animals (main effect of age, $p<0.001$ ). ${ }^{\text {bb-ee }}$

\section{Negative geotaxis}

LC-treated pups took longer to reorient themselves than VEH-treated controls (main effect of treatment, $p=$ 0.001 ), and the latency to reorient decreased with age (main effect of age, $p<0.001$; Fig. 3f). ${ }^{\text {ff-ii }}$

\section{Locomotion}

We detected a significant treatment $\times$ age interaction $(p<0.001$; Fig. 3g). Post hoc comparisons found that LC-treated pups displayed increased locomotion as early as PN10 $(p<0.001)$, which persisted through PN14 $(p<$ 0.001 for PN11, $p=0.004$ for PN12, $p<0.001$ for PN13, $p<0.001$ for PN14). ${ }^{\mathrm{j} j-\mathrm{ww}}$

\section{Neonatal prosocial behaviors \\ Nest-seeking task}

LC-treated pups performed significantly worse in the nest-seeking task, successfully locating and reaching the goal arm containing their home bedding fewer total times ( $p<0.001 ;$ Fig. $4 b)$. LC-treated pups reached the goal arm fewer times at all ages tested (main effect of treatment, $p<0.001$; Fig. $4 a$ ), although the ability to seek the nest improved with age for both groups (main effect of age, $p=0.008$ ). The latency to reach a goal arm significantly decreased with age regardless of treatment (main effect of age, $p<0.001$; Fig. $4 c$ ). ${ }^{\mathrm{x}-\mathrm{ggg}}$ Thus, it is unlikely that the observed impairments in performance were due to locomotor differences between groups.

\section{Maternal isolation-induced USVs}

LC-treated pups emitted significantly fewer USVs than VEH-treated controls when isolated from the dam $(p<$

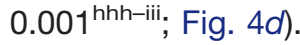

\section{Juvenile memory-dependent tasks}

Spontaneous alternations

LC-treated rats successfully alternated between arms significantly fewer times than VEH-treated rats $p=$ 0.026 $6^{\mathrm{ijj}-\mathrm{kkk}}$; Fig. 5a).
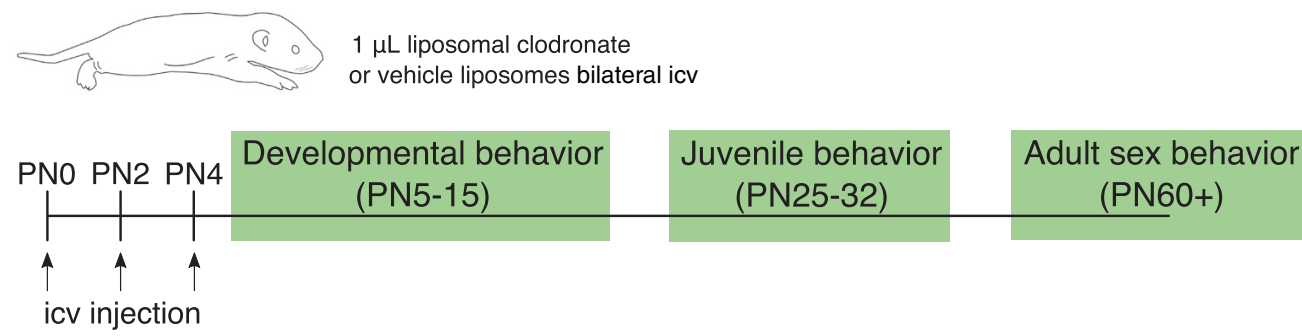

Figure 1. Treatment and behavioral testing timeline. Schematic depicting the ages at which treatments and behavioral testing were performed. 


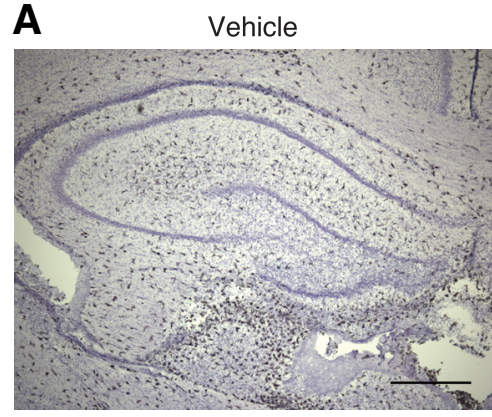

D

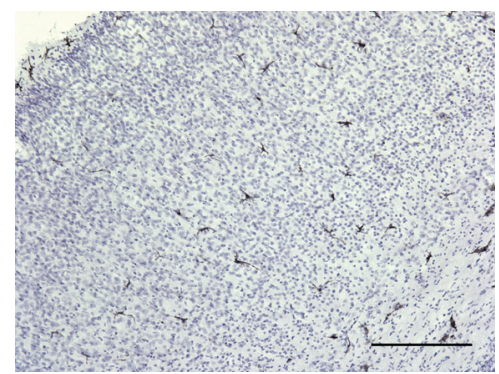

G

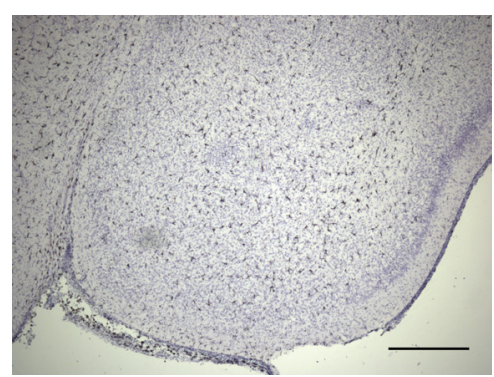

J

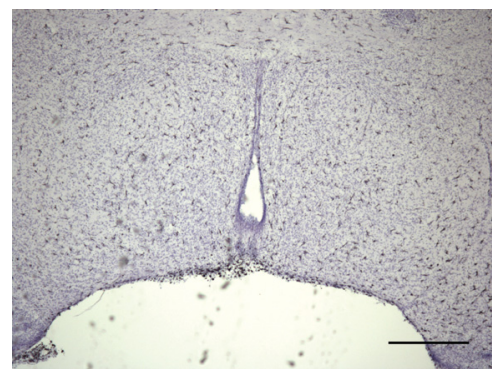

B

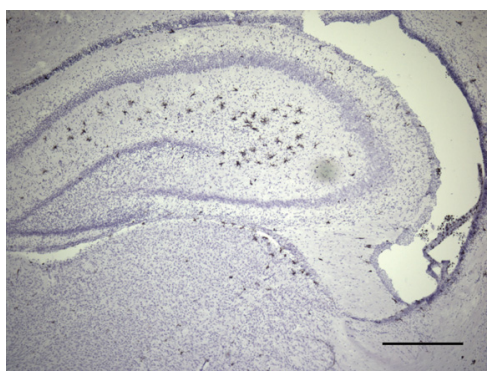

E

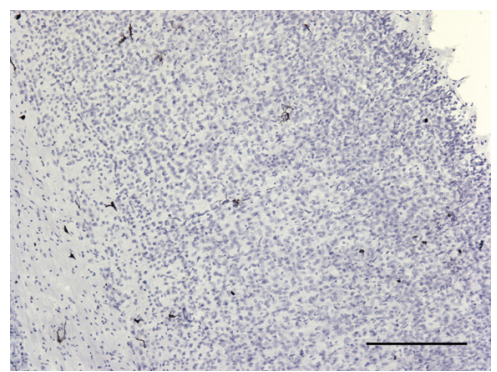

H

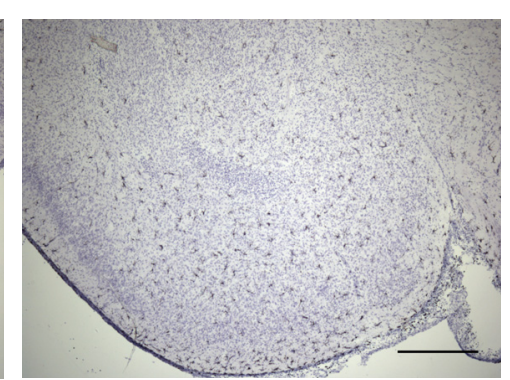

K

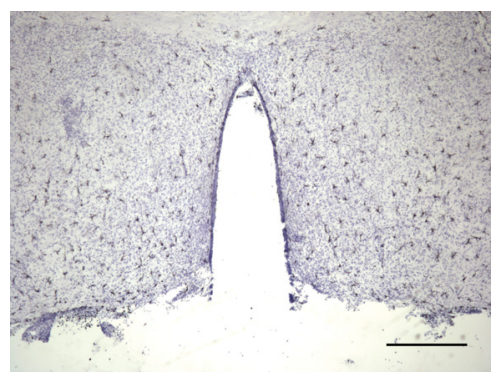

C

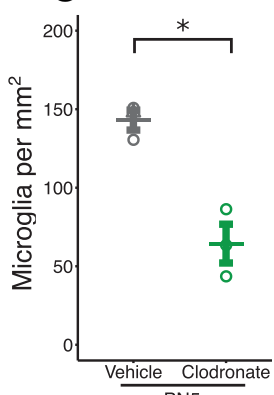

F
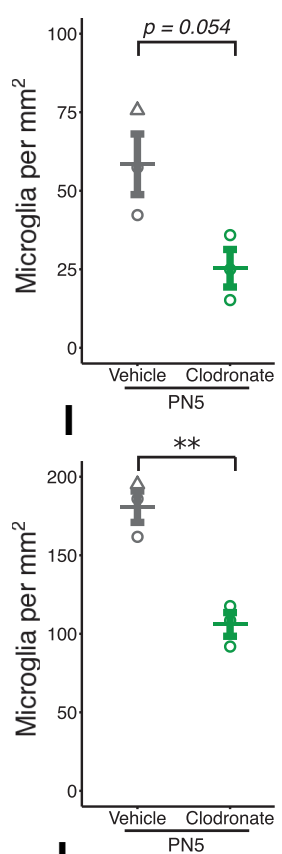

L

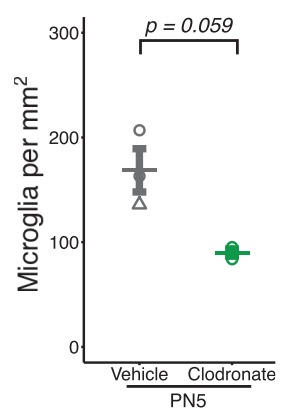

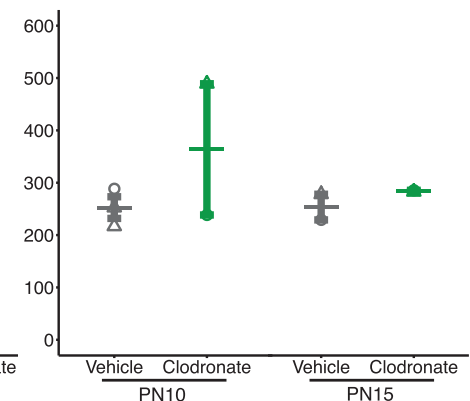
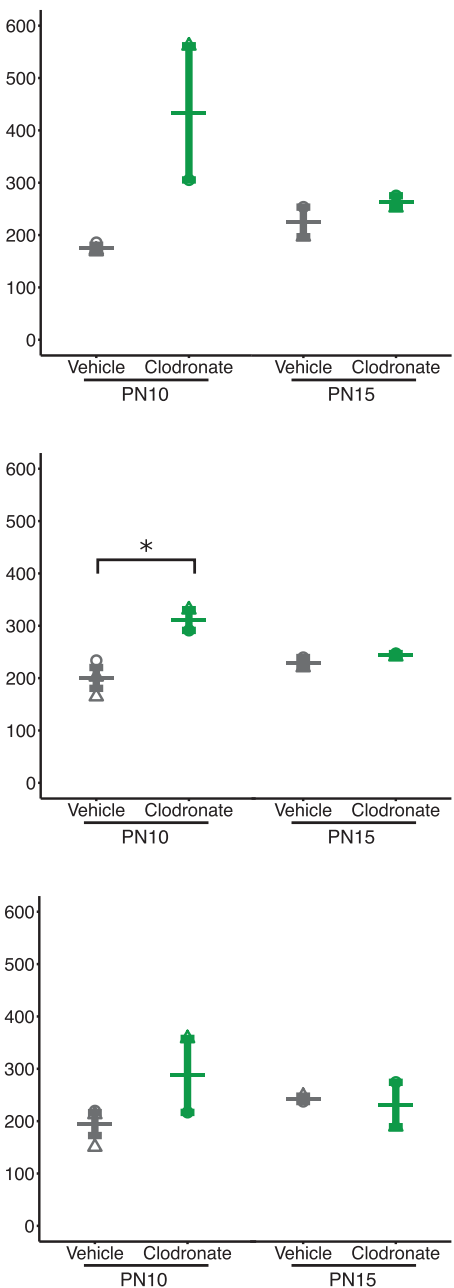

Figure 2. Microglia numbers are reduced after clodronate treatment and rapidly repopulate. Coronal sections of brains from PN5 VEH-treated $(\boldsymbol{a}, \boldsymbol{d}, \boldsymbol{g}, \boldsymbol{j})$ and LC-treated $(\boldsymbol{b}, \boldsymbol{e}, \boldsymbol{h}, \boldsymbol{k})$ rats. Representative images from sections labeled for Iba1 and counterstained with hematoxylin from the hippocampus $(\boldsymbol{a}, \boldsymbol{b})$, cortex $(\boldsymbol{d}, \boldsymbol{e})$, amygdala $(\boldsymbol{g}, \boldsymbol{h})$, and hypothalamus $(\boldsymbol{j}, \boldsymbol{k})$. Quantification of $\mathrm{lba} 1^{+}$cell density in the hippocampus (c), cortex (f), amygdala (i) , and hypothalamus (I) at PN5, 10, and 15. Scale bars $=500 \mu \mathrm{m}(\boldsymbol{a}, \boldsymbol{b}, \boldsymbol{g}, \boldsymbol{h}, \boldsymbol{j}, \boldsymbol{k})$ or 250 $\mu \mathrm{m}(\boldsymbol{d}, \boldsymbol{e})$. Bars indicate group means \pm SEM for VEH-treated (black) and LC-treated (green) rats of both sexes. Open circles indicate data from individual female rats, and triangles, from male rats. $* p<0.05, * * p<0.01 . n=2-3$ per treatment.

\section{Social recognition}

VEH-treated rats displayed intact recognition memory, indicated by a recognition index significantly greater than chance $(p=0.003 ; 0.50=$ chance level). LC-treated rats failed to recognize the familiar stimulus rat with a recognition index equal to chance $(p=$ 0.863); however, direct comparison between groups did not reach statistical significance $(p=0.083$; Fig. 5b). ${ }^{111-0 o 0}$

\section{Novel object recognition}

Initial analysis found a strong trend for an effect of sex on novel object preference at $1 \mathrm{~h}(p=0.059$; data not shown), as males appeared to have an increased 


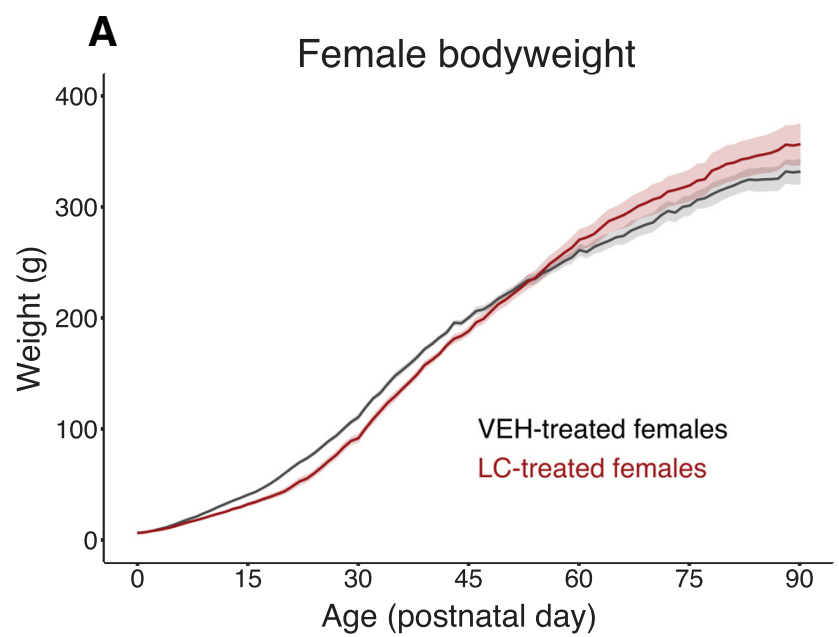

VEH-treated
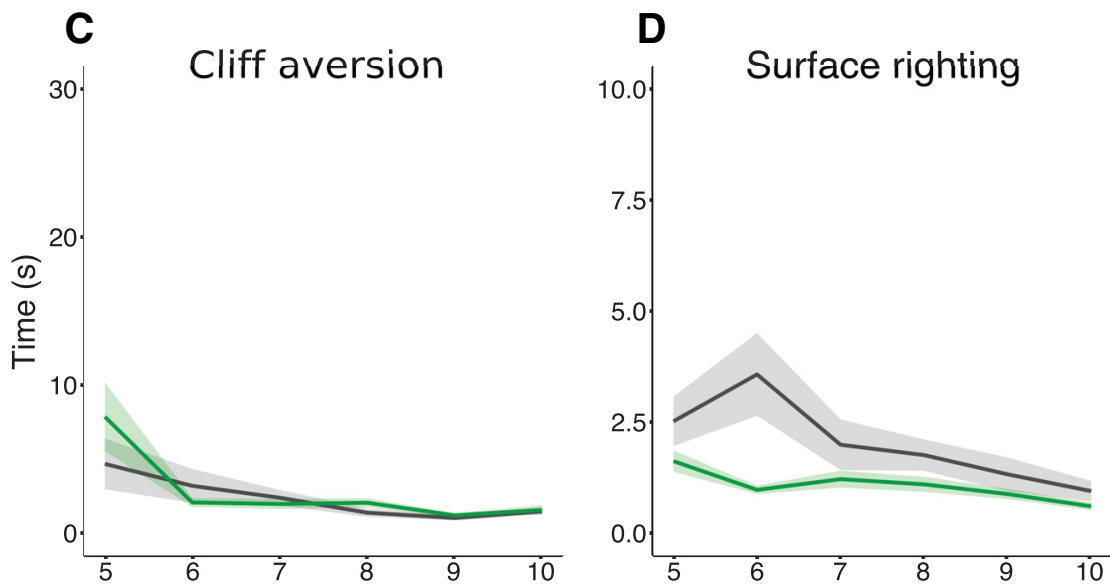

\section{Male bodyweight}

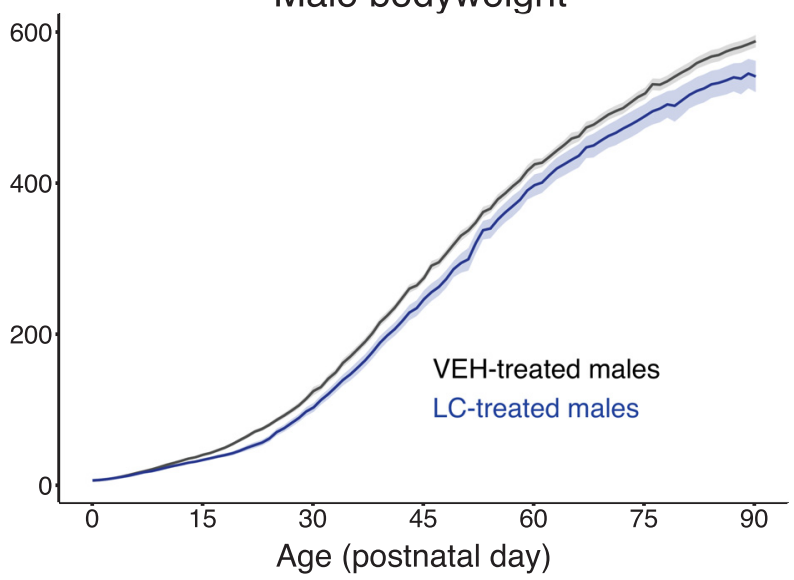

LC-treated

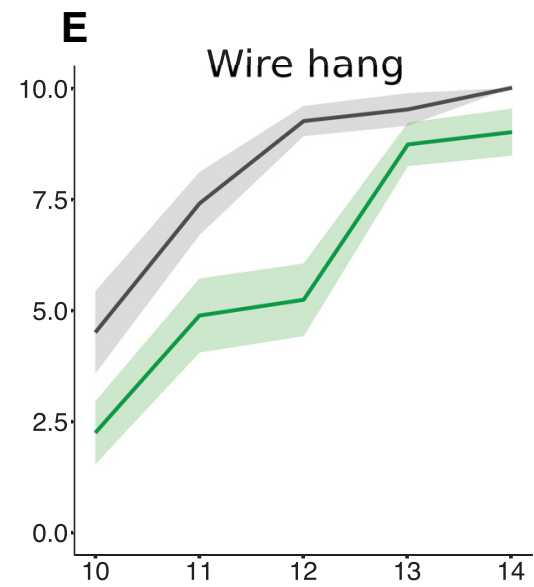

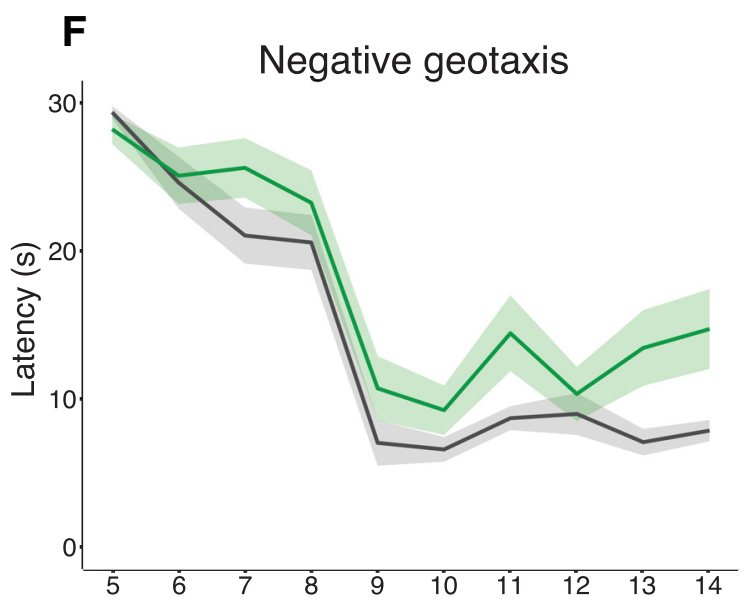

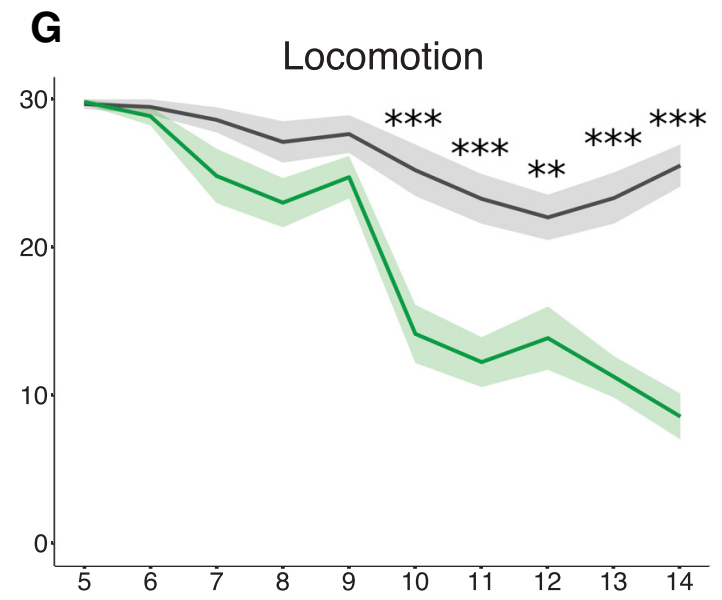

Figure 3. Microglia depletion alters the development of early reflexes, behaviors, and body weight throughout life. Body weight (in grams) in female (a) and male (b) rats measured daily from PNO to PN90. $\boldsymbol{c}$, Time (in seconds) for rat pups to retreat from an edge (cliff aversion). $\boldsymbol{d}$, Time (in seconds) for rat pups to successfully right themselves (surface righting). e, Duration (in seconds) rat pups hung from their forepaws before falling (wire hang). $\boldsymbol{f}$, Latency (in seconds) for rat pups to reverse orientation and face upward on an incline (negative geotaxis). $\boldsymbol{g}$, Latency (in seconds) for rat pups to leave a designated area (locomotion). Solid lines indicate group means for VEH-treated (black; $\boldsymbol{a}-\boldsymbol{g}$ ) and LC-treated (red; $\boldsymbol{a})$ females, LC-treated males (blue; $\boldsymbol{b}$ ), and LC-treated rats of both sexes (green; $\boldsymbol{c}-\boldsymbol{g}$ ). Shaded regions indicate \pm SEM. $* * p<0.01$, $* * * p<0.001 . n=18-20$ per treatment. 
A
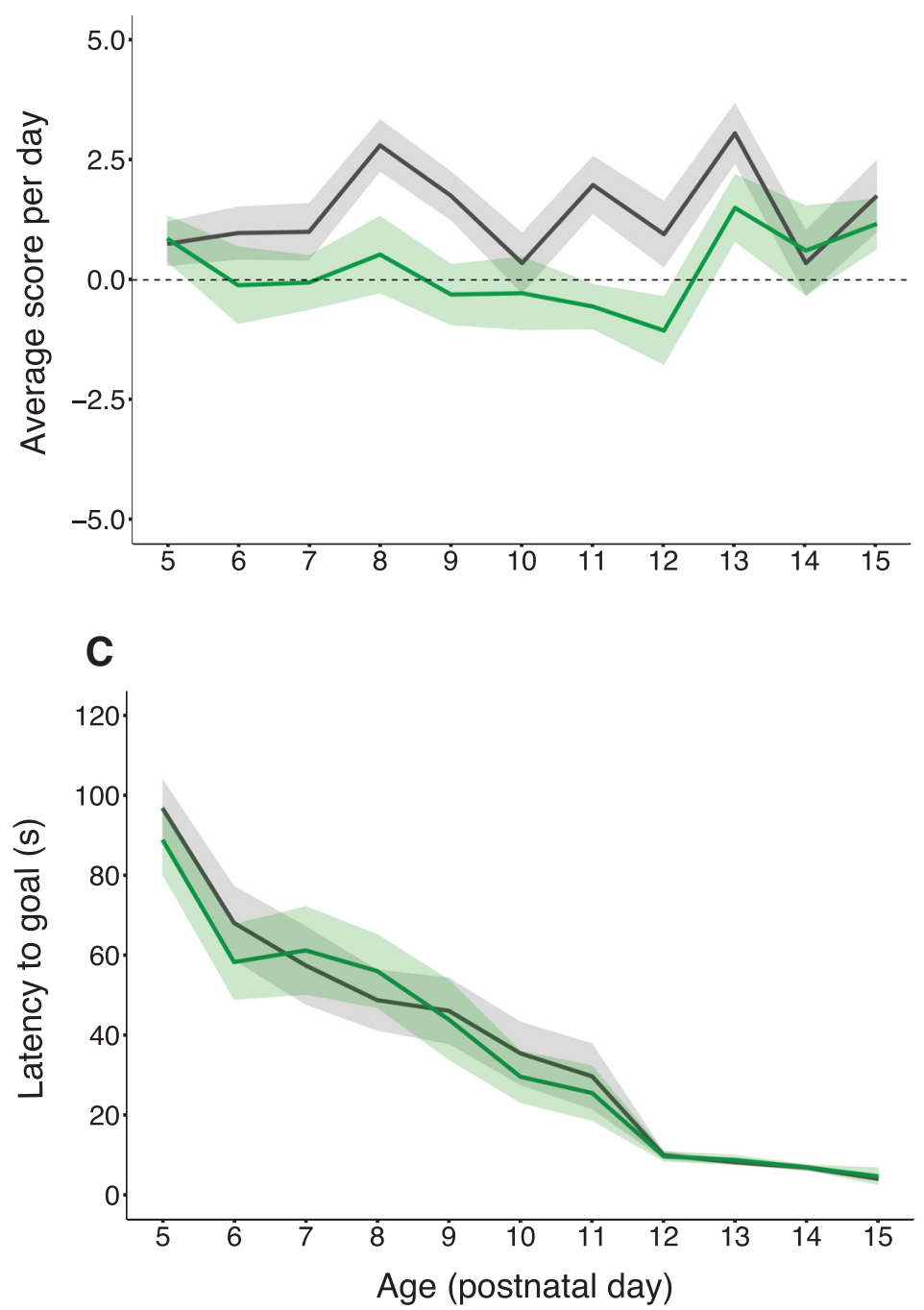
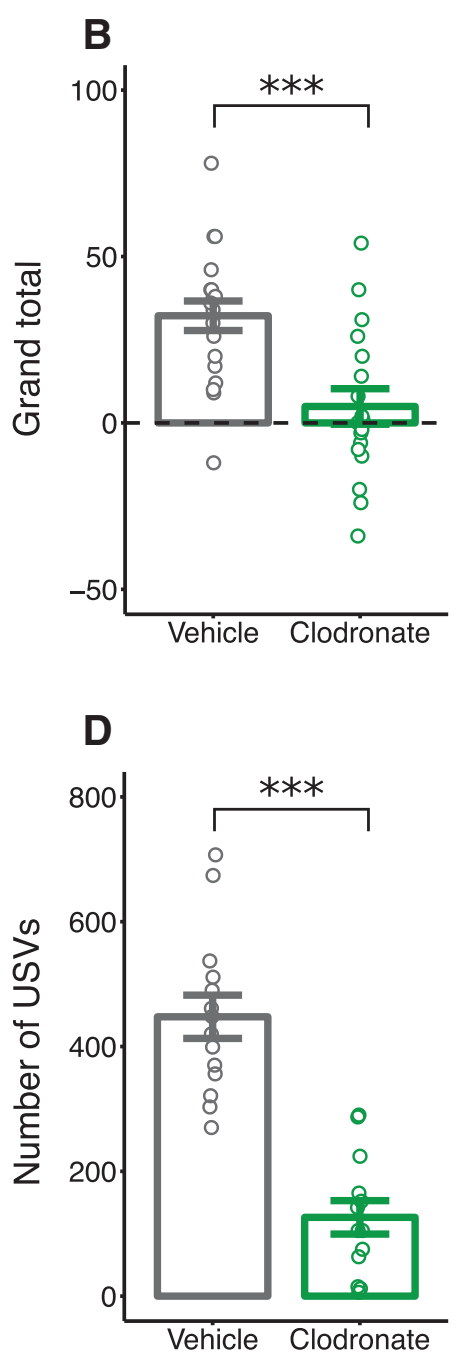

Figure 4. Postnatal microglia depletion impairs nest-seeking behavior and decreases USV emission. Quantification of data from the two-choice nest-seeking task represented as average score per day (a), average total score summed across all testing days (b), and average latency (in seconds) to reach either goal arm of the testing apparatus (c). $\boldsymbol{d}$, Quantification of the number of USVs produced by pups when separated from the mother on PN8. Solid lines and bars indicate group means for VEH-treated (black) and LC-treated (green) rats of both sexes. Shaded regions and error bars indicate \pm SEM. Open circles represent data from individual rats. Horizontal dashed line indicates score equal to chance $(\boldsymbol{a}, \boldsymbol{b})$. $* * * p<0.001 . n=18-20(\boldsymbol{a}-\boldsymbol{c})$ and $13-14$ (d) per treatment.

recognition index. The same analysis found no effect of sex at $24 \mathrm{~h}$ ( $p=0.673$; data not shown). After collapsing the data across sex, we found no difference between groups in recognition index at $1 \mathrm{~h}(p=0.907$; Fig. $5 c)$, but did detect a trend toward a reduction in recognition index for LC-treated rats after a $24-\mathrm{h}$ retention interval $(p=$ 0.094). ppp-sss $^{\text {pro }}$

\section{Juvenile locomotor and anxiety-like behavior Open field}

LC-treated rats displayed less anxiety-like behavior, spending significantly more time in the center of an open field $(p=0.006$; Fig. $6 a)$. Moreover, they exhibited a hyperlocomotive phenotype evidenced by an increased number of line crossings in the open field compared with VEH-treated rats $(p<0.001$; Fig. $6 b) .{ }^{\mathrm{tt}-\mathrm{www}}$

\section{Elevated plus maze}

Similar to the open field test, LC-treated rats spent significantly more time in the open arms compared with VEH-treated controls $\left(p<0.001^{\text {xxx-yyy}} ;\right.$ Fig. $\left.6 c\right)$.

\section{Juvenile fear, avoidance, and risk-assessing behaviors}

In the predator odor exposure test, LC-treated rats spent less time behind the barrier-a measure of behavioral avoidance-compared with VEH-treated controls (main effect of treatment, $p=0.002$ ), and both groups spent more time behind the barrier in response to cat odor (main effect of test phase, $p=0.018$; Fig. 7a). LC-treated 
A

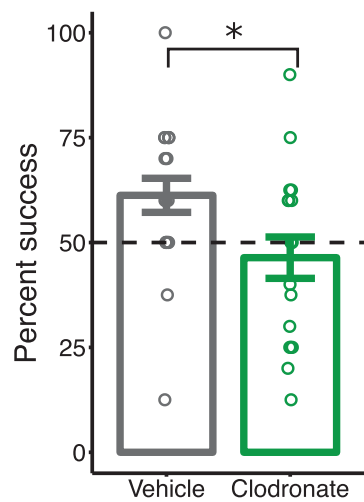

T-maze Alternations
B

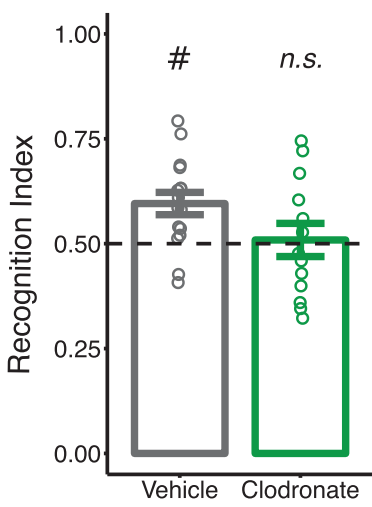

Social Recognition

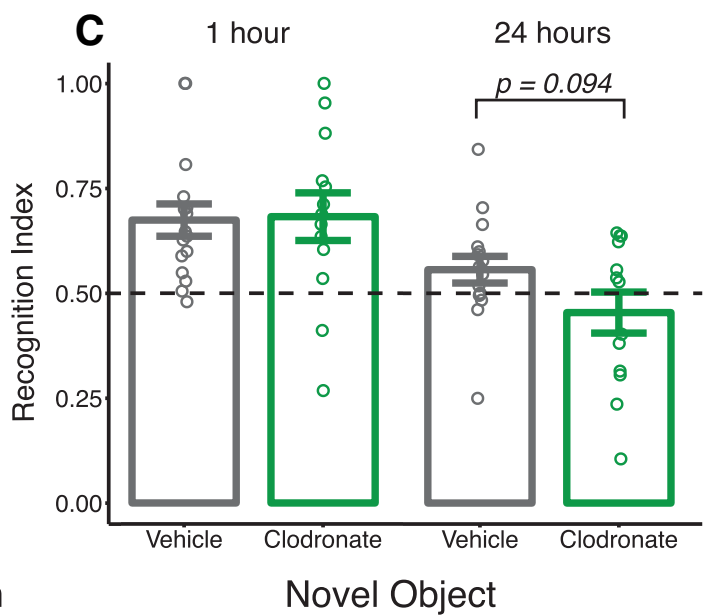

Figure 5. Postnatal microglia depletion induces selective memory impairment in juvenile rats. a, Percentage successful spontaneous alternations in a T-maze from PN25 to PN29. $\boldsymbol{b}$, Social recognition index calculated on PN30 after a $30-$ min retention interval. c, Novel object recognition index calculated after a 1-h (PN26) and 24-h (PN27) retention interval. Bars indicate group means \pm SEM for VEH-treated (black) and LC-treated (green) rats of both sexes. Open circles represent data from individual rats. Horizontal dashed line indicates score equal to chance $(a-c) . * p<0.05$. \#p $<0.01$ compared with chance. $n=$ 18-20 (a) and 13-16 (b, c) per treatment.

rats appeared to spend less time freezing across test phases; however, this effect did not reach statistical significance (main effect of treatment, $p=0.065$; Fig. $7 b$ ). We found treatment $\times$ test phase interactions for two measures of risk-assessing behaviors: stretch-attend duration $(p=0.013$; Fig. $7 c)$ and stretch-locomotion duration ( $p<0.001$; Fig. $7 d$ ). Post hoc analysis revealed that VEH-treated rats increased the amount of time engaged in stretch-attend behavior when exposed to cat odor ( $p<$ 0.001 for VEH), which was near significant for LC-treated rats $\left(p=0.038, \alpha_{\text {adjusted }}=0.025\right)$. LC-treated rats spent less time in stretched attendance than VEH-treated rats while exposed to cat odor ( $p=0.005)$, with no baseline differences between groups. Interestingly, LC-treated rats spent more time engaged in stretched locomotion while exposed to cat odor ( $p=0.002$ compared to baseline), and significantly more compared with VEH-treated controls $(p<0.001)$, with no baseline differences between groups. Overall, LC-treated rats approached the stimulus cloth more than VEH-treated rats (main effect of treatment, $p=0.007$ ), and the number of approaches decreased for both groups when the cloth was scented with cat odor (main effect of test phase, $p<0.001$; Fig. 7e). Accordingly, LC-treated rats spent more time interacting with the stimulus cloth across both test phases (main effect of treatment, $p<0.001$; Fig. $7 f$ ). ${ }^{\text {zzz-eeee }}$
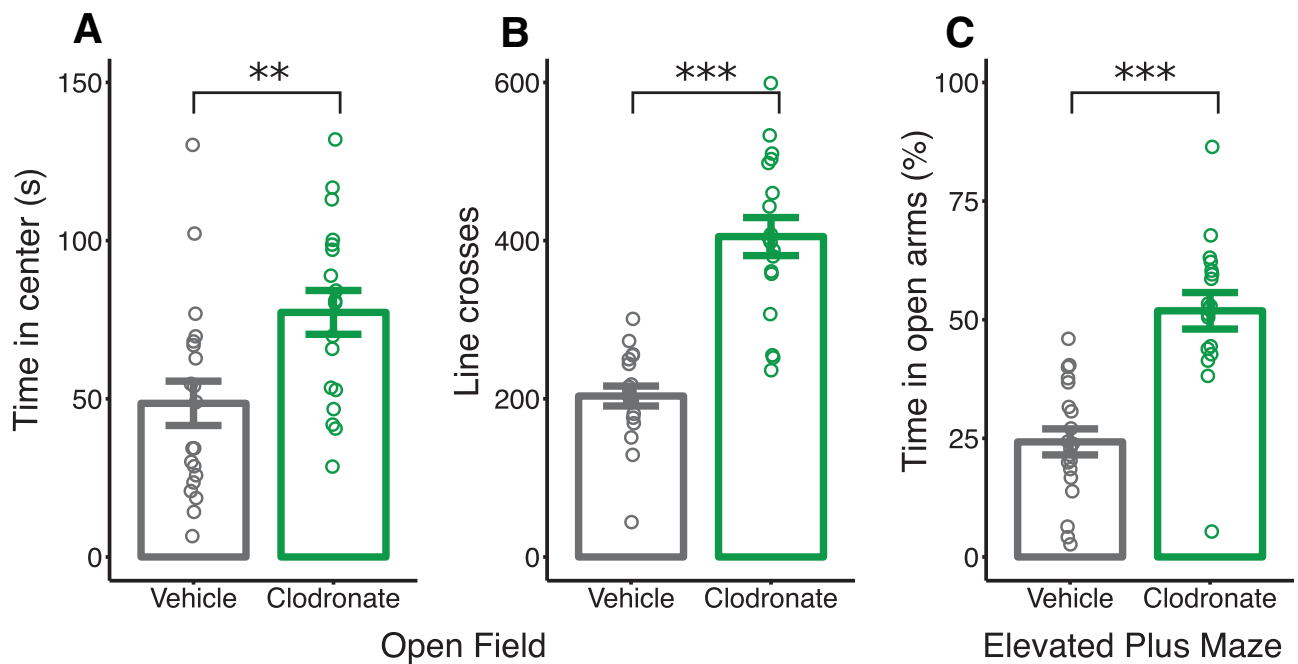

Figure 6. Postnatal microglia depletion increases locomotion, center time in the open field, and open arm time in the elevated plus maze in juvenile rats. Center time (a; in seconds) and gridline crosses (b) in an open field arena on PN25. $\boldsymbol{c}$, Percentage of time in the open arms of an elevated plus maze on PN32. Bars indicate group means \pm SEM for VEH-treated (black) and LC-treated (green) rats of both sexes. Open circles represent data from individual rats. $* * p<0.01, * * * p<0.001 . n=18-20$ per treatment. 
A
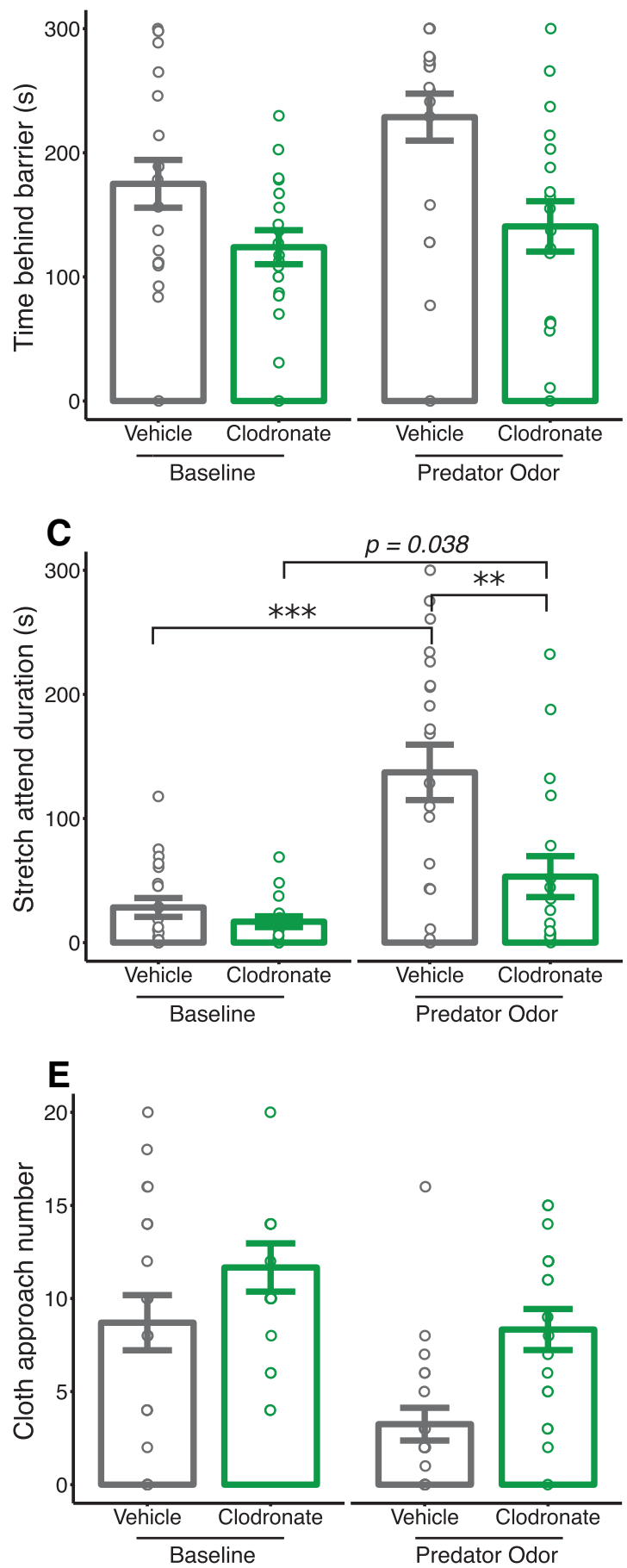

B
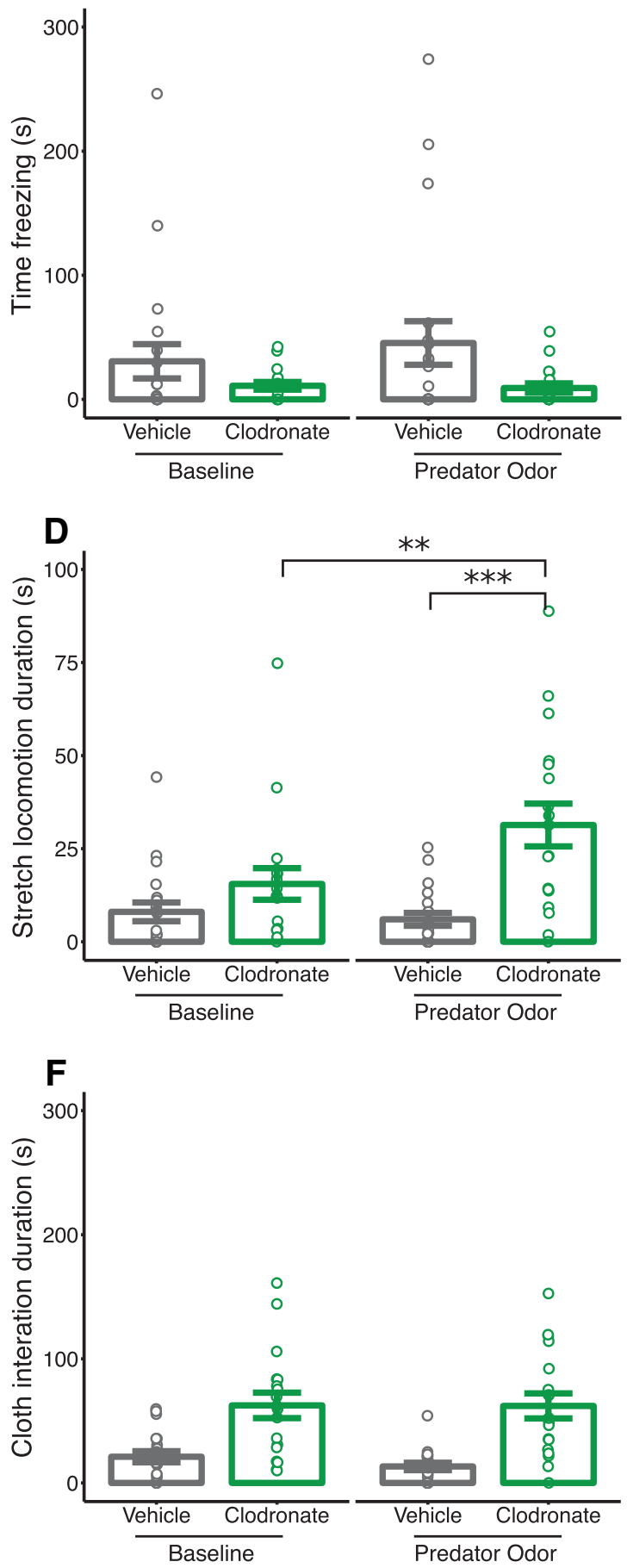

Figure 7. Microglia-depleted animals exhibit decreased fear and avoidance but display risk-assessing behaviors. Quantification of data from the predator odor exposure test (PN33) for time (in seconds) spent in various behaviors including time behind the barrier (a), freezing behavior (b), stretch-attend behavior (c), stretch-locomotion behavior (d), number of stimulus cloth approaches $(\boldsymbol{e})$, and stimulus cloth interaction duration $(\boldsymbol{f})$. Bars indicate group means \pm SEM for VEH-treated (black) and LC-treated (green) rats of both sexes. Open circles represent data from individual rats. $* p<0.05, * * p<0.01, * * * p<0.001$. $n=18-20$ per treatment.

\section{Adult sex behavior}

As microglia are critical to the masculinization of male sex behavior during the early postnatal period (Lenz et al., 2013), we analyzed adult male and female rats for the expression of sex behaviors. Accordingly, we predicted that LC-treated males would display impaired sex behavior, whereas female behavior would be unaffected by clodronate treatment. 
A

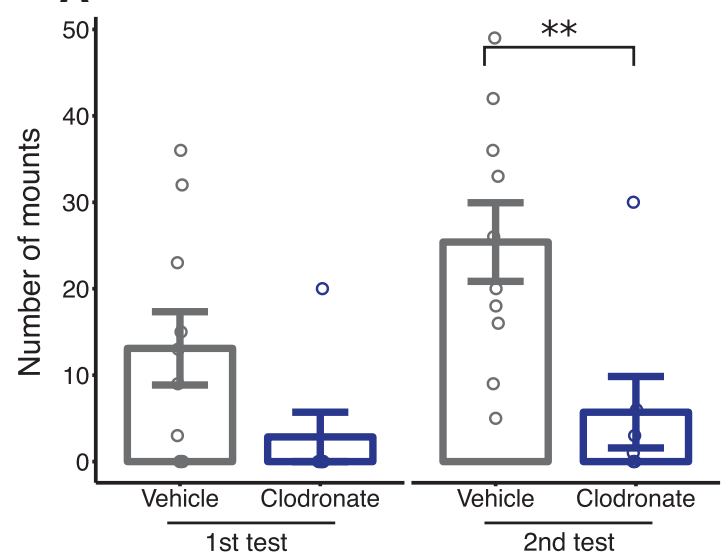

C

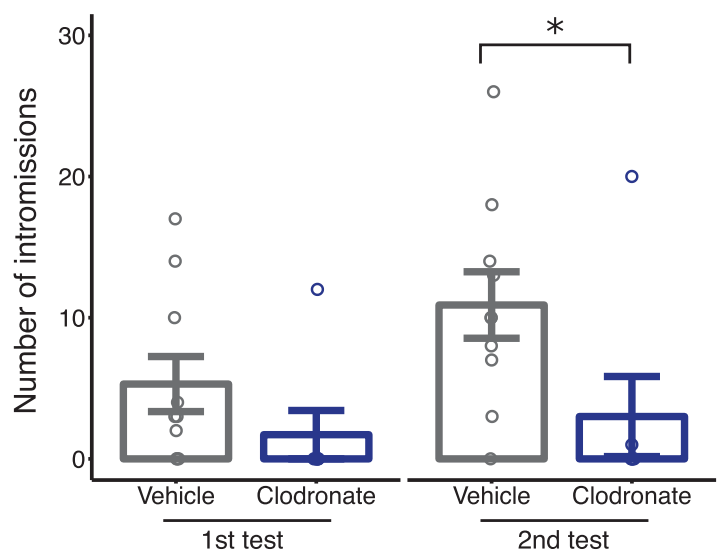

E

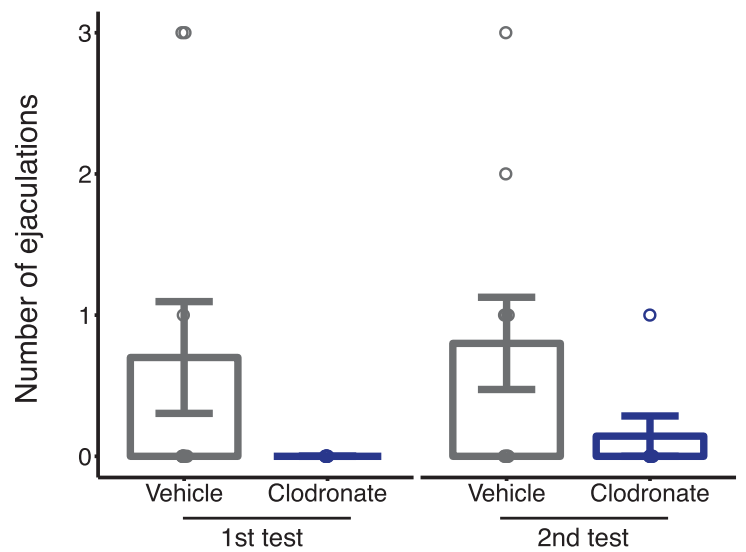

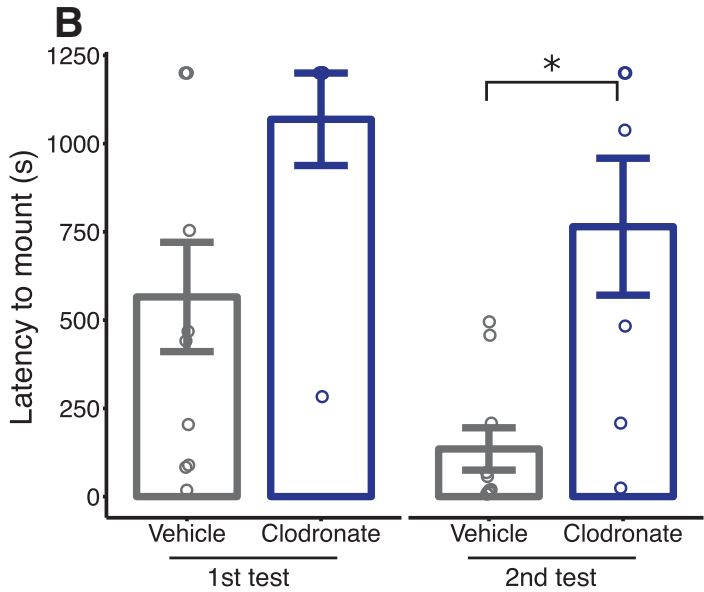

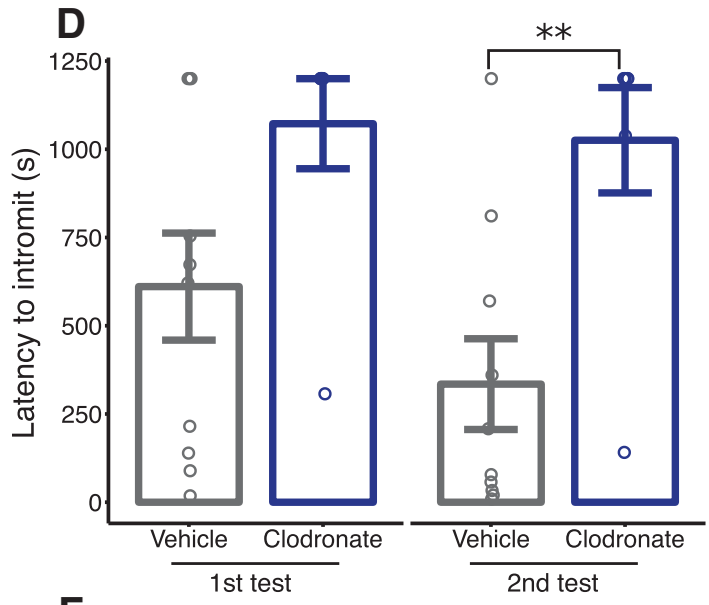

$\mathbf{F}$

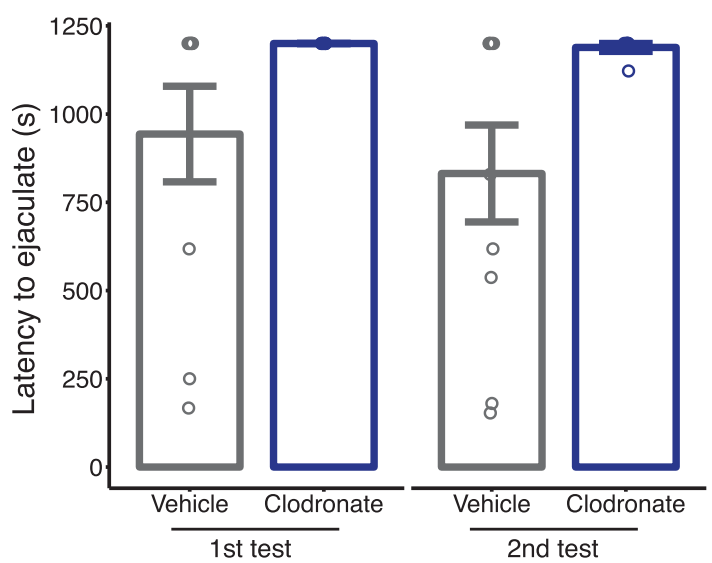

Figure 8. Postnatal microglia depletion induces deficits in adult male sex behaviors. Quantification of various components of male sex behavior including number of mounts (a), latency to first mount (b), number of intromissions (c), latency to first intromission $(\boldsymbol{d})$, number of ejaculations $(\mathbf{e})$, and latency to first ejaculation $(\boldsymbol{f})$. Solid bars indicate group means \pm SEM for VEH-treated (black) and LC-treated (blue) male rats. Open circles represent data from individual rats. $* p<0.05, * * p<0.01$. $n$ $=7-10$ per treatment.

\section{Male sex behavior}

Consistent with our predictions, LC-treated males mounted fewer times than VEH-treated males on the second test day $(p=0.007$; Fig. $8 a)$. This corresponded to a significantly longer latency to first mount in LC-treated males $(p=0.011 ;$ Fig. $8 b)$. Similarly, LC-treated males intromitted fewer times ( $p=0.028$; Fig. $8 c$ ), with an increased latency to first intromission $(p=0.009$; Fig. $8 d$ ). We did not detect a difference in the expression of ejaculatory behaviors $(p=0.134$; Fig. $8 e$ ) or the latency to first 
A

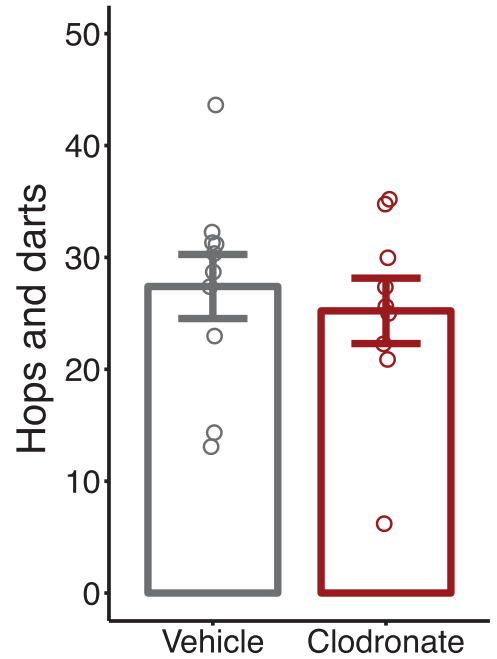

B

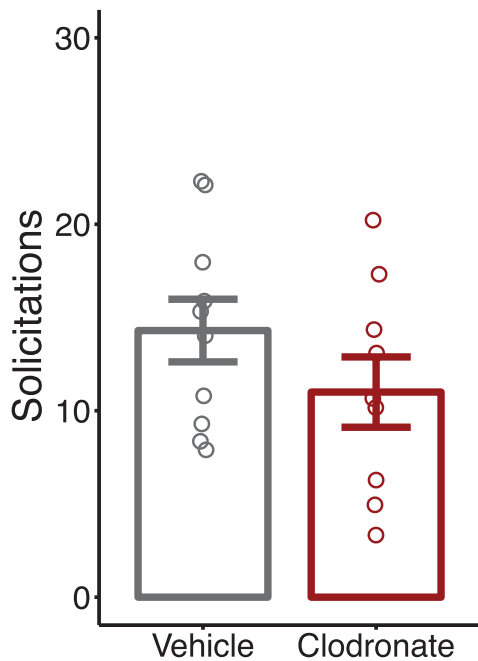

C

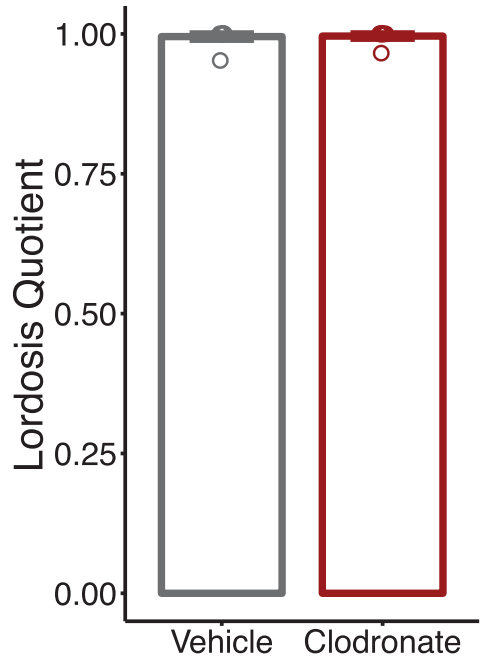

Figure 9. Adult female sex behavior is unchanged after postnatal microglia depletion. Quantification of female sexual proceptivity: number of hops and darts (a) and solicitations $(\boldsymbol{b})$ and receptivity lordosis quotient $(\boldsymbol{c})$. Solid bars indicate group means \pm SEM for VEH-treated (black) and LC-treated (red) female rats. Open circles represent data from individual rats. $n=9-10$ per treatment.

ejaculation ( $p=0.098$; Fig. $8 f$ ) between treatments, although this is likely due to the low incidence of ejaculation during our tests. fffff-kkkkk

\section{Female sex behavior}

We found no difference in the expression of proceptive behaviors (hops and darts, $p=0.602$, Fig. 9a; solicitations, $p=0.209$, Fig. $9 b$ ) or receptivity as measured by the lordosis quotient ( $p=1$; Fig. $9 c$ ) between treatments. IIIII-nnnnn

\section{Factor analysis of juvenile behaviors}

We performed factor analysis on various outcomes from the juvenile behavioral battery to identify potential underlying behavioral constructs that might help explain the patterns of behavior we observed. Our analysis identified four factors to be sufficient to explain our data, accounting for $66.6 \%$ of total variance (Fig. 10a). The first factor (19.4\% of total variance) contained positive loadings for performance on the elevated plus maze, predator odor object interaction duration, and stretch-locomotion and negative loadings for predator odor stretch attend duration and freezing duration. As the behaviors enriched in factor 1 largely reflected measures of behavioral disinhibition, we renamed it as such ("Disinhibition"). Similarly, the second factor (named "Locomotion"; $17.0 \%$ of total variance) contained positive loadings for open-field line crosses, open-field center time, predator odor stretchlocomotion, and negative loading for T-maze alternations, all behaviors reflecting locomotion and exploration. The third factor ("Risk Assessment"; 16.6\% of total variance) included positive loadings for time behind the barrier and stretched attend duration in the predator odor test, and the fourth factor ("Cognitive Association"; $13.7 \%$ of variance) included positive loadings for predator odor freezing duration and social recognition index.

To determine whether these factors differed between sexes or treatment groups, we calculated factor scores for each subject. Comparisons between males and females found no significant differences for any of the four factors; however, comparisons between treatments found significantly higher scores for LC-treated rats in factor 1 ( $p$ $=0.003)$ and factor $2(p<0.001)$ compared with VEHtreated rats, with no differences in scores detected for either factor 3 or factor 4 (Fig. 10b).

\section{Discussion}

Here we report lasting alterations in the expression of neonatal, juvenile, and adult behaviors after transient microglia depletion in the developing rat brain. These effects are apparent in early life, as both male and female rat pups displayed markedly deficient pro-social behaviors in addition to a pervasive hyperlocomotive phenotype. In juvenile animals, the consequences of early microglia depletion cross multiple behavioral domains and include working memory deficits and alterations in the expression of fear, anxiety-like, and risk-assessment behaviors. Adult rats show sex-specific sensitivity to early microglia depletion, exhibiting differential outcomes in body weight and male-specific impairments in sex behaviors.

The most remarkable of our findings is the immediate and lasting impact of transient microglia depletion on brain development and behavior. We observed perturbations in brain architecture as early as PN10, evidenced by the appearance of enlarged ventricles and a thinned cortex. These effects are likely attributable to microglia, as liposomal clodronate selectively depletes macrophages, via apoptosis, while sparing nonphagocytic cells (van Rooijen et al., 1996; van Rooijen and Hendrikx, 2010), and this technique has been used to deplete microglia without affecting other cell types (Faustino et al., 2011; Torres et al., 2016).

Similar alterations in gross morphology have been reported in CSF-1R knockout mice that display a $>99 \%$ reduction in microglia throughout embryonic and postna- 

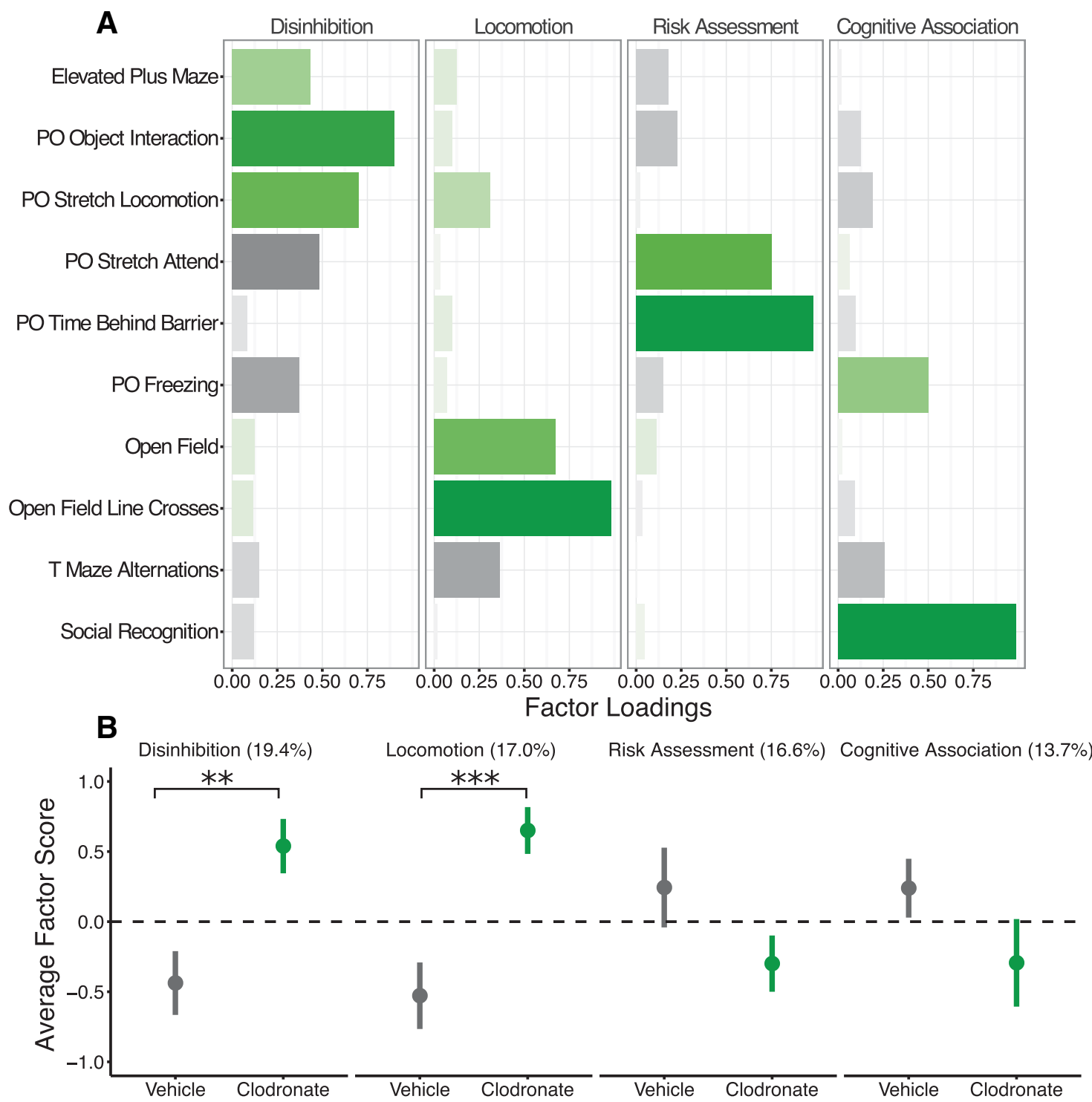

Figure 10. Factor analysis results. (a) Behavior factor loadings for each named factor. Longer, saturated bars indicate stronger loadings, with green indicating positive loading and black indicating negative loading. (b) Loading scores for VEH and LC rats plotted for each factor. The values in parentheses represent the percentage of total variance accounted for by each factor. Bars indicate group means \pm SEM for VEH-treated (black) and LC-treated (green) rats of both sexes. $* * p<0.01, * * * p<0.001 . n=13-16$ per treatment.

tal development. Brain development in CSF-1R knockout mice appears to proceed normally during embryonic development, with abnormalities most prominent at 3 weeks of age (Erblich et al., 2011). Our findings suggest a much shorter window for the manifestation of these effects, as by $\mathrm{PN} 10$, rapid regrowth restored microglia numbers to levels comparable to controls. Moreover, $24 \mathrm{~h}$ after the last clodronate injection (PN5), we observed a $\sim 50 \%$ rate of depletion in many brain regions. It is especially striking, then, that modest reductions in microglia over a short period of time produce such profound brain abnormalities and major alterations in behavior. Perhaps equally notable, the rats in our study appeared to live healthy lives into adulthood, and the behavioral data had consistently small variance, similar to that of control rats despite large deviation in brain development.
Microglia are critical to the development of the embryonic and postnatal brain (Frost and Schafer 2016), yet data connecting early microglia function to behavioral maturation is currently lacking. One such study, published during the preparation of this manuscript, used a similar approach to address this issue (Nelson and Lenz, 2016). Our findings expand on those, as we used a behavioral battery to assess the appropriate acquisition and development of pups' motor reflexes, strength, and coordination with the aim of identifying early markers of dysfunction. From this battery, we identified a hyperlocomotive phenotype that persisted through the juvenile period. Previous reports find no changes in locomotion with various microglia manipulations in adult mice (Rogers et al., 2011; Elmore et al., 2014; Torres et al., 2016); thus, it is likely that our findings reflect a novel developmental role for 
microglia in mediating the maturation of locomotor behavior.

The neonatal period of life also marks the development of early pro-social behaviors. We used a nest-seeking task and measured maternal isolation-induced USVs. The nest-seeking task measures pups' ability to identify and locate the maternal nest and depends on the display of adequate olfactory, motor, and discriminatory abilities to discern nest bedding from clean bedding (Gregory and Pfaff, 1971), whereas pup USVs are instructional to the dam to elicit attention and retrieval. Together, these two behaviors represent two ethologically relevant and important aspects of the pup side of the maternal-pup relationship that are often disrupted in models of neurodevelopmental disorders (Branchi et al., 2001). Consistent with the notion of an early social deficit, microglia-depleted pups demonstrated no preference for the maternal bedding and emitted fewer isolation-induced USVs than their undepleted littermates. We did not determine whether maternal behavior toward pups was altered in those in which the microglia were depleted; additional studies will need to investigate the nature of mother-infant interactions as they pertain to the early, and perhaps later, social deficits reported here.

Others have reported effects of microglia depletion in mature mice on various memory-dependent tasks, social deficits, and conditioned fear responses. We find similar deficiencies across these behavioral domains in juvenile animals. Specifically, microglia-depleted rats had fewer successful spontaneous alternations, which may reflect impairments in working memory, but this response may also be interpreted as a broader measure of cognitive inflexibility or repetitive behavioral patterning. Despite these deficiencies, we did not find any difference in novel object preference, suggesting that microglia-depleted rats were capable of integrating and retaining information for prolonged periods of time.

Microglia-depleted rats also displayed impaired social memory. Taken together with our findings in the nestseeking task, these data suggest pervasive disruptions in the processing of social information. Conspecific recognition is one aspect of a social interaction, and further studies are needed to examine whether the observed social impairments extend to more complex social behaviors. Along these lines, a recent report in rats found that neonatal microglia depletion induced minor deficits in juvenile play behavior and increased social avoidance in adulthood (Nelson and Lenz, 2016), consistent with our findings here.

There are conflicting reports on the role of microglia in anxiety-like behaviors (Rogers et al., 2011; Elmore et al., 2015). When assessing performance in the open field and elevated plus maze, we found that microglia-depleted juvenile rats exhibited a drastic reduction in anxiety-like behaviors. Given the hyperlocomotive phenotype in the open field, this may reflect a measure of behavioral disinhibition or impulsivity, rather than a measure of anxiety. To this point, in pilot studies and the data presented here, nearly $20 \%$ of microglia-depleted animals jumped off of the elevated plus maze during the test and continued to explore the testing room. One such animal ended the test in this manner in fewer than $5 \mathrm{~s}$. Our findings are strikingly similar to those of Nelson and Lenz (2016), who found increased locomotion and decreased anxiety-like behavior in the open field and elevated plus maze, even observing comparable numbers of animals that jumped off the elevated plus maze open arms.

Rodents freeze in response to many fear-evoking stimuli. Others have reported reductions in freezing behavior in response to fear conditioning in mouse lines with genetic depletion of microglia (Rogers et al., 2011; Parkhurst et al., 2013). Fear conditioning requires the formation of a learned association between a particular cue (context or specific stimulus) and a negative outcome. To avoid the confounding effects of differences in learning, we used an assay to examine innate fear that involved a predator odor. We observed a decrease in avoidance behaviors in microglia-depleted juveniles and detected a strong trend toward a decrease in freezing behavior. As our testing arena contained an opaque barrier for the animals to retreat behind, it is likely that the magnitude of any freezing behavior was diminished and animals engaged in other defensive behaviors. Interestingly, microglia depletion induced opposite effects on measures of risk assessment. Microglia-depleted rats displayed more stretchattend behavior and stretched locomotion toward the predator odor. These data together suggest that although the overt expression of fear and avoidance is decreased in microglia-depleted rats, they retain the ability to recognize and assess a potential threat and adjust their behavior accordingly. These findings are consistent with the notion of a behaviorally disinhibited phenotype, rather than a decrease in general anxiety-like behavior.

Whereas males and females were equally affected in our developmental battery and juvenile tests of memory, fear, and anxiety, the two sexes were differentially affected with respect to sexually dimorphic sex behaviors. To this point, we found male-specific deficits in the expression of sex behavior and not in the expression of female sex behavior. These findings corroborate those of Lenz et al., (2013), who found microglia to be a key mediator of the masculinization of sex behaviors. Our understanding of how microglia may mediate organizational sex differences in the brain is still in its infancy, but interestingly, many of the mechanisms by which sex differences are established are also developmental processes that are heavily influenced by microglia (Lenz and McCarthy, 2015; McCarthy et al., 2015). Further investigation into how microglia may act to sculpt sexually dimorphic circuitry in the brain is critical to advancing our understanding of a fundamental aspect of brain development.

A common theme throughout our behavioral data was the presence of a hyperlocomotive and behaviorally disinhibited phenotype observed in LC rats. Our factor analysis supports this conclusion, identifying four factors, two of which were significantly different between treatment groups. The first factor related to performance in the elevated plus maze, and stretch locomotion, stretch attend, cloth interactions, and freezing behavior in the 
predator odor assay. Together, this factor may reflect behavioral disinhibition across contexts. The second factor related to time in the center of an open field, open field grid crosses, predator odor stretch locomotion, and T maze alternations, which together may be representative of locomotion and exploratory behavior. We speculate that the effects of developmental microglia depletion affect the maturation of brain circuitry underlying these behavioral constructs. The exact cellular and circuit level manifestation of our findings remains a subject for further investigation.

\section{References}

Alliot F, Godin I, Pessac B (1999) Microglia derive from progenitors, originating from the yolk sac, and which proliferate in the brain. Brain Res Dev Brain Res 117:145-152. Medline

Antony JM, Paquin A, Nutt SL, Kaplan DR, Miller FD (2011) Endogenous microglia regulate development of embryonic cortical precursor cells. J Neurosci Res 89:286-298. CrossRef

Bessis A, Béchade C, Bernard D, Roumier A (2007) Microglia control of neuronal death and synaptic properties. Glia 55:233-238. CrossRef Medline

Branchi I, Santucci D, Alleva E (2001) Ultrasonic vocalisation emitted by infant rodents: a tool for assessment of neurobehavioural development. Behav Brain Res 125:49-56. CrossRef

Cunningham CL, Martínez-Cerdeño V, Noctor SC (2013) Microglia regulate the number of neural precursor cells in the developing cerebral cortex. J Neurosci 33:4216-4233. CrossRef Medline

Davalos D, Grutzendler J, Yang G, Kim JV, Zuo Y, Jung S, Littman DR, Dustin ML, Gan WB (2005) ATP mediates rapid microglial response to local brain injury in vivo. Nat Neurosci 8:752-758. CrossRef Medline

Elmore MR, Najafi AR, Koike MA, Dagher NN, Spangenberg EE, Rice RA, Kitazawa M, Matusow B, Nguyen H, West BL, Green KM (2014) Colony-stimulating factor 1 receptor signaling is necessary for microglia viability, unmasking a microglia progenitor cell in the adult brain. Neuron 82:380-397. CrossRef

Elmore MR, Lee RJ, West BL, Green KM (2015) Characterizing newly repopulated microglia in the adult mouse: impacts on animal behavior, cell morphology, and neuroinflammation. PLoS One 10: e0122912 CrossRef

Erblich B, Zhu L, Etgen AM, Dobrenis K, Pollard JW (2011) Absence of colony stimulating factor-1 receptor results in loss of microglia, disrupted brain development and olfactory deficits. PLoS One 6:e26317 CrossRef Medline

Faustino JV, Wang X, Johnson CE, Klibanov A, Derugin N, Wendland MF, Vexler ZS (2011) Microglial cells contribute to endogenous brain defenses after acute neonatal focal stroke. J Neurosci 31: 12992-13001. CrossRef Medline

Frost JL, Schafer DP (2016) Microglia: architects of the developing nervous system. Trends Cell Biol 26:587-597. CrossRef Medline

Ginhoux F, Greter M, Leboeuf M, Nandi S, See P, Gokhan S, Mehler MF, Conway SJ, Ng LG, Stanley ER, Samokhvalov IM, Merad M (2010) Fate mapping analysis reveals that adult microglia derive from primitive macrophages. Science 330:841-845. CrossRef Medline

Gregory EH, Pfaff DW (1971) Development of olfactory-guided behavior in infant rats. Physiol Behav 6:573-576. Medline

Hoshiko M, Arnoux I, Avignone E, Yamamoto N, Audinat E (2012) Deficiency of the microglial receptor CX3CR1 impairs postnatal functional development of thalamocortical synapses in the barrel cortex. J Neurosci 32:15106-15111. CrossRef Medline

Ji K, Akgul G, Wollmuth LP, Tsirka SE (2013) Microglia actively regulate the number of functional synapses. PLoS One 8:e56293 CrossRef Medline

Kettenman H, Hanisch UK, Noda M, Verkhratsky A (2011) Physiology of microglia. Physiol Rev 91:461-553. CrossRef Medline

Kierdorf K, Erny D, Goldmann T, Sander V, Schulz C, Perdiguero EG, Wieghofer P, Heinrich A, Riemke P, Hölscher C, Müller DN,
Luckow B, Brocker T, Debowski K, Fritz G, Opdenakker G, Diefenbach A, Biber K, Heikenwalder M, Geissmann F, Rosenbauer F, Prinz M (2013) Microglia emerge from erythromyeloid precursors via Pu.1- and Irf8-dependent pathways. Nat Neurosci 16:273-280. CrossRef

Lenz KM, Nugent BM, Haliyur R, McCarthy MM (2013) Microglia are essential to masculinization of brain and behavior. J Neurosci 33:2761-2772. CrossRef Medline

Lenz KM, McCarthy MM (2015) A starring role for microglia in brain sex differences. Neuroscientist 21:306-321. CrossRef Medline

Marín-Teva JL, Dusart I, Colin C, Gervais A, van Rooijen N, Mallat M (2004) Microglia promote the death of developing Purkinje cells. Neuron 41:535-547. Medline

McCarthy MM, Pickett LA, VanRyzin JW, Kight KE (2015) Surprising origins of sex differences in the brain. Hormones Behav 76:3-10. CrossRef Medline

Nelson LH, Lenz KM (2016) Microglia depletion in early life programs persistent changes in social, mood-related, and locomotor behavior in male and female rats. Behav Brain Res 316:279-293. CrossRef Medline

Nimmerjahn A, Kirchhoff F, Helmchen F (2005) Resting microglial cells are highly dynamic surveillants of brain parenchyma in vivo. Science 308:1314-1318. CrossRef Medline

Nikodemova M, Kimyon RS, De I, Small AL, Collier LS, Watters JJ (2015) Microglial numbers attain adult levels after undergoing a rapid decrease in cell number in the third postnatal week. J Neuroimmunol 278:280-288.

Morgan SC, Taylor DL, Pocock JM (2004) Microglia release activators of neuronal proliferation mediated by activation of mitogenactivated protein kinase, phosphatidylinositol-3-kinase/Akt and delta-Notch signalling cascades. J Neuroimmunol 90(1):89-101.

Paolicelli RC, Bolasco G, Pagani F, Maggi L, Scianni M, Panzanelli P, Giustetto M, Ferreira TA, Guiducci E, Dumas L, Ragozzino D, Gross CT (2011) Synaptic pruning by microglia is necessary for normal brain development. Science 333:1456-1458. CrossRef Medline

Parkhurst CN, Yang G, Ninan I, Savas JN, Yates JR, Lafaille JJ, Hempstead BL, Littman DR, Gan WB (2013) Microglia promote learning-dependent synapse formation through brain-derived neurotrophic factor. Cell 155:1596-1609. CrossRef Medline

R Core Team (2016) R: A language and environment for statistical computing. R Foundation for Statistical Computing, Vienna, Austria.

Rogers JT, Morganti JM, Bachstetter AD, Hudson CE, Peters MM, Grimmig BA, Weeber EJ, Bickford PC, Gemma C (2011) CX3CR1 deficiency leads to impairment of hippocampal cognitive function and synaptic plasticity. J Neurosci 31:16241-16250. CrossRef Medline

Schafer DP, Lehrman EK, Kautzman AG, Koyama R, Mardinly AR, Yamasaki R, Ransohoff RM, Greenberg ME, Barres BA, Stevens B (2012) Microglia sculpt postnatal neural circuits in an activity- and complement-dependent manner. Neuron 74:691-705. CrossRef

Schwarz JM, Sholar PW, Bilbo SD (2012) Sex differences in microglia colonization of the developing rat brain. J Neurochem 120:948963. CrossRef Medline

Shigemoto-Mogami Y, Hoshikawa K, Goldman JE, Sekino Y, Sato K (2014) Microglia enhance neurogenesis and oligodendrogenesis in the early postnatal subventricular zone. J Neurosci 34:2231-2243. CrossRef Medline

Sierra A, Encinas JM, Deudero JJ, Chancey JH, Enikolopov G, Overstreet-Wadiche LS, Tsirka SE, Maletic-Savatic M (2010) Microglia shape adult hippocampal neurogenesis through apoptosiscoupled phagocytosis. Cell Stem Cell 7:483-495. CrossRef Medline

Stevens B, Allen NJ, Vazquez LE, Howell GR, Christopherson KS, Nouri N, Micheva KD, Mehalow AK, Huberman AD, Stafford B, Sher A, Litke AM, Lambris JD, Smith SJ, John SW, Barres BA (2007) The classical complement cascade mediates CNS synapse elimination. Cell 131(6):1164-1178. 
Squarzoni P, Oller G, Hoeffel G, Pont-Lezica L, Rostaing P, Low D, Bessis A, Ginhoux F, Garel S (2014) Microglia modulate wiring of the embryonic forebrain. Cell Rep 8:1271-1279. CrossRef Medline

Torres L, Danver J, Ji K, Miyauchi JT, Chen D, Anderson ME, West BL, Robinson JK, Tsirka SE (2016) Dynamic microglial modulation of spatial learning and social behavior. Brain Behav Immun 55:6-16.

Tremblay ME, Lowery RL, Majewska AK (2010) Microglial interactions with synapses are modulated by visual experience. PLoS Biol 8:e100527. CrossRef Medline

Ueno M, Fujita Y, Tanaka T, Nakamura Y, Kikuta J, Ishii M, Yamashita $T$ (2013) Layer $V$ cortical neurons require microglia support for survival during postnatal development. Nat Neurosci 16:543-551. CrossRef van Rooijen N, Sanders A, van den Berg TK (1996) Apoptosis of macrophages induced by liposome-mediated intracellular delivery of clodronate and propamidine. J Immunol Methods 193(1):93-9 van Rooijen N, Hendrikx E (2010) Liposomes for specific depletion of macrophages from organs and tissues. Methods Mol Biol 605: 189-203. CrossRef Medline

Wakselman S, Béchade C, Roumier A, Bernard D, Triller A, Bessis A (2008) Developmental neuronal death in hippocampus requires the microglial CD11b integrin and DAP12 immunoreceptor. J Neurosci 28:8138-8143. CrossRef Medline

Zhan Y, Paolicelli RC, Sforazzini F, Weinhard L, Bolasco G, Pagani F, Vyssotski AL, Bifone A, Gozzi A, Ragozzino D, Gross CT (2014) Deficient neuron-microglia signaling results in impaired functional brain connectivity and social behavior. Nat Neurosci 17:400-406. CrossRef Medline 